AVERAGE CROSSING TIME:

\title{
AN ALTERNATIVE CHARACTERIZATION OF MEAN AVERSION AND REVERSION
}

\author{
John B. Donaldson \\ Rajnish Mehra \\ Working Paper 25519 \\ http://www.nber.org/papers/w25519 \\ NATIONAL BUREAU OF ECONOMIC RESEARCH \\ 1050 Massachusetts Avenue \\ Cambridge, MA 02138 \\ January 2019
}

We thank Parantap Basu and George Constantinides for their helpful comments and Tyler Beason, Seunghoon $\mathrm{Na}$ and Sergio Villar for programming assistance. The views expressed herein are those of the authors and do not necessarily reflect the views of the National Bureau of Economic Research.

NBER working papers are circulated for discussion and comment purposes. They have not been peer-reviewed or been subject to the review by the NBER Board of Directors that accompanies official NBER publications.

(C) 2019 by John B. Donaldson and Rajnish Mehra. All rights reserved. Short sections of text, not to exceed two paragraphs, may be quoted without explicit permission provided that full credit, including (O) notice, is given to the source. 
Average Crossing Time: An Alternative Characterization of Mean Aversion and Reversion John B. Donaldson and Rajnish Mehra

NBER Working Paper No. 25519

January 2019

JEL No. C13,C53,E3,E44,E47,G1,G12

\begin{abstract}
$\underline{\text { ABSTRACT }}$
We evaluate the properties of mean reversion and mean aversion in asset prices and returns as commonly characterized in the finance literature. The study is undertaken within a class of wellknown dynamic stochastic general equilibrium models and shows that the mean reversion/ aversion distinction is largely artificial. We then propose an alternative measure, the 'Average Crossing Time' that both unifies these concepts and provides an alternative characterization. Ceteris paribus, mean reverting processes have a relatively shorter average crossing time as compared to mean averting processes.
\end{abstract}

John B. Donaldson

Columbia Business School

3022 Broadway, Uris Hall

New York, NY 10027

jd34@gsb.columbia.edu

Rajnish Mehra

Department of Economics

W. P. Carey School of Business

Arizona State University

PO Box 879801

Tempe, AZ 85287-9801

and NBER

rajnish.mehra@asu.edu 


\section{Introduction}

There has been a long-standing debate in the asset pricing literature as to whether time series of equity and bonds returns are 'mean reverting' or 'mean averting'. While the conventional wisdom is that the former returns are mean reverting and the latter mean averting, the issue is by no means settled. ${ }^{1}$ The debate has also diffused to the predictability literature. The implicit underlying belief in predictability studies is that the predicting variables (dividend-price ratios, earnings-price ratios) follow stationary processes that revert to some unspecified normal value (the mean of the process). As Campbell and Shiller (2001) put it: "It seems reasonable to suspect that prices are not likely ever to drift too far from their normal levels relative to indicators of fundamental value, ... when stock prices are very high relative to these indicators, then prices will eventually fall in the future to bring the ratios back to more normal historical levels". Intuitively, a mean reverting process captures this property: above average values of the stochastic process must regularly be followed by below average values and vice-versa. Conversely, mean aversion intuitively suggests that the stochastic process drifts away from its mean and implies that mean aversion is unlikely to be a characteristic of a stationary time series. We show that this intuition is misleading.

\footnotetext{
${ }^{1}$ There is a plethora of empirical studies on mean reversion in stock prices at various time horizons. See for example, papers by Summers (1986), Campbell and Mankiw (1987), Fama and French (1988), Lo and MacKinlay (1988) and Poterba and Summers (1988). Others, most notably Kim, Nelson and Startz (1991) and Richardson and Stock (1989) have challenged some of their conclusions. See also the conflicting perspectives in, e.g., Lewellen (2004), Torous et al. (2004), and Campbell and Yogo (2006) versus Goyal and Welch (2003), Welch and Goyal (2008) and Bossaerts and Hillion (1999). Other important work includes Cochrane(2011), Kim and Nelson (1998), Bessembinder et al. (1995) and Daniel (2001). Zakamulin (2015), provided an excellent summary of this literature and explores the evidence for mean reversion and predictability over periods exceeding ten years.
} 
There are at least four characterizations of mean reversion in the finance literature and it is our intention to evaluate their relationship vis-à-vis the above intuition. Multiple characterizations of mean reversion (aversion) have arisen, in part, because the empirical literature is hampered by the insufficiency of financial time series of data for discriminating statistical tests. We sidestep this issue by considering stochastic model economies that allow the generation of arbitrarily long return series. Specifically, we call into service several widely used dynamic production-based macro-finance models, where the time series of security prices and their returns are stationary by construction, and explore the extent to which they satisfy these various characterizations. A key finding for these models is that the measures of mean reversion/aversion used in the literature classify some of these series as mean reverting and others as mean averting.

Negative (positive) autocorrelation in financial return series, for example, is sometimes cited as the identifying characteristic of mean reversion (aversion) and we adopt it as our benchmark property. In particular, this identifying characteristic classifies the stationary time series characterizing equity returns as 'mean reverting' yet the stationary time series characterizing bond returns is mean averting (a result consistent with conventional understanding) in the case of the simplest baseline equilibrium model. This result demonstrates that mean reversion, as defined by the benchmark property, is not unique to the phenomenon that 'above average values of stochastic process must regularly be followed by below average values and vice-versa' since mean averting time series are equally consistent with this characterization. We later show that more realistic macroeconomic versions of the model generically display benchmark mean aversion in all financial time series, whether equity or debt, a result that leads us to question the usefulness of that characterization.

In this paper we propose an alternative measure, "Average Crossing Time" $(A C T)$ to characterize mean reversion/aversion. We argue that it provides not only a more useful measure of the degree of mean reversion/aversion, but also a 
more intuitive sense of one time series being "more strongly meanreverting/averting' than another. It also allows us to rank existing characterizations of mean reversion according to how restrictive their measure of mean reversion actually is.

As its name suggests, the $A C T$ measures the average number of periods in the evolution of a discrete time stochastic process that the process is strictly above or below its mean value. Broadly speaking, mean reverting processes have a relatively shorter average crossing time as compared to mean averting ones. Using the $A C T$ measure we explore the antecedent probability structures behind the traditional notions of mean reversion and aversion. We identify the properties of the $A C T$ measure and compare them with the traditional autocorrelation measures for the class of macroeconomic models referred to above. Our analysis is both analytical and computational, with the latter consisting of wide-ranging numerical simulations.

An outline of the paper is as follows: In section 2 we identify four definitions of mean reversion and mean aversion found in the literature, and partially characterize their interrelationships. In Section 3, these definitions are applied to the analysis of a simple baseline dynamic macroeconomic model. ${ }^{2}$ The $A C T$ characterization of "mean reversion" is introduced in Section 4. In Section 5 we add additional features to the Baseline model and study the resulting implications for the strength of mean reversion/aversion in model-generated

\footnotetext{
${ }^{2}$ The primary intellectual antecedents of the present study are Basu and Vinod (1994), Cecchetti et al. (1990), Guvenen (2009), and Lansing (2015). In a Lucas (1978) style exchange model where dividends follow a Markov switching regime (see Hamilton (1989)), Cecchetti et al. (1990) are able to replicate the observed patterns of mean reversion measures at various horizons. Guvenen (2009) explores asset pricing in a model where firm owners and workers have differential access to securities markets: firm owners trade both equity and default free bonds while workers are limited to bond trading. Lansing (2015) explores the asset pricing consequences of variation in factor shares. Both report slight negative correlation in equity returns based on data, and as equilibrium outcomes of their models. We note that the analysis in Basu and Vinod (1994) explores some of the same issues and motivates the present study. None of these studies, however, explores the fundamental information being conveyed by the mean reversion / aversion property, but rather focus on identifying this property in model generated return series.
} 
equity returns and equity premium data. Section 6 relates these concepts to the data by computing empirical autocorrelations and $A C T \mathrm{~s}$ for prominent financial return series while Section 7 concludes.

\section{Mean Reversion}

The empirical finance literature proposes multiple characterizations of "mean reversion." In the discussion below we examine these alternate characterizations and explore their interrelationships. They are as follows, expressed in terms of an arbitrary stationary stochastic process $\left\{\tilde{x}_{t}\right\}$.

A stationary stochastic process $\left\{\tilde{x}_{t}\right\}$ is said to be mean reverting if and only if:

I.

$$
\begin{aligned}
& \text { I. } \quad \operatorname{cov}\left(\tilde{x}_{t}, \tilde{x}_{t+1}\right)<0 \\
& \text { II. } \quad \frac{\operatorname{var}\left(\tilde{x}_{t}+\tilde{x}_{t+1}+\ldots+\tilde{x}_{t+j}\right)}{j+1}<\operatorname{var} \tilde{x}_{t}, \text { for any } j \geq 1 .
\end{aligned}
$$

Property I is cited by Guvenen (2009) and Lansing (2015). An early proponent of Property II is Summers (1986). Property II is also used in Poterba and Summers (1988) and Mukherji (2011) for their discussions of mean reversion in stock price and rate of return series. ${ }^{3}$

The relationship between Properties I and II is captured in Proposition 2.1.

Proposition 2.1: Let $\left\{\tilde{x}_{t}\right\}$ be a stationary, mean reverting, stochastic process with an ergodic probability distribution. With respect to that distribution, statistical properties I and II detailed above are related according to

a) $\quad$ II $\Rightarrow$ I

\footnotetext{
${ }^{3}$ Property II stands in specific contrast to the analogous property of a random walk where $\operatorname{var}\left(\tilde{x}_{t}+\tilde{x}_{t-1}+\ldots+\tilde{x}_{t+j-1}\right)=j \operatorname{var}\left(\tilde{x}_{t}\right)$.
} 
b) If $\left|\operatorname{cov}\left(\tilde{x}_{t}, \tilde{x}_{t+1}\right)\right|>\left|\sum_{s=2}^{j-1} \operatorname{cov}\left(\tilde{x}_{t}, \tilde{x}_{t+s}\right)\right|$ for all $j$

then $\quad$ I $\Rightarrow$ II. $^{4}$

Proof: See the Technical Appendix.

Condition (3) captures the idea that a process displays strong comovement in adjacent elements but that the effect diminishes very rapidly for series elements progressively in the future.

The significance of Proposition 2.1, simple as it is, lies in Part a): if the financial series generated by a particular model fails to satisfy Property I, it will fail to satisfy Property II as well. In this sense Property II is a more restrictive criterion than Property I. For this reason we focus on assessing how restrictive Property I actually is, knowing that Property II is even more so. In fact, they will be shown by the $A C T$ measure to be so strikingly restrictive that it is not surprising to find they are not robustly satisfied in data.

III. For any time integers $0 \leq r<s<t<u$,

$$
\operatorname{cov}\left(x_{s}-x_{r}, x_{u}-x_{t}\right)<0 .
$$

Property III, to our knowledge first proposed in Exley et al. (2004), is a comment about sequential changes in the values of the stochastic process $\left\{\tilde{x}_{t}\right\}$ rather than a statement about the statistical properties of the values themselves. Interpreting $\tilde{x}_{t}$ as $\tilde{p}_{t}^{e}$, the price of equity (capital) at time $t$, Property III suggests that increases in the price of equity over a particular interval of time will generally be followed by reductions in the price in future time intervals. As such, it represents a sense of mean reversion different from Properties I and II. In all of

\footnotetext{
${ }^{4}$ For mean aversion the connections of Proposition 2.1 are reversed. Using the notation of Proposition 2.1 they are:

a) $\mathrm{I} \Rightarrow \mathrm{II}$

b) If $\left|\operatorname{cov}\left(\tilde{x}_{t}, \tilde{x}_{t+1}\right)\right|<\left|\sum_{s=2}^{j-1} \operatorname{cov}\left(\tilde{x}_{t}, \tilde{x}_{t+s}\right)\right|$ for all $j$ then II $\Rightarrow \mathrm{I}$. 
the characterizations we consider, if the identifying inequality is reversed, the series is said to be mean averting.

Property III can be guaranteed if certain sufficient conditions are satisfied.

For any time integers $h>0, k>0$, define $v(h-k)$ as:

$$
v(h-k) \equiv \operatorname{var}\left(\tilde{x}_{h}-\tilde{x}_{k}\right)
$$

This allows a simple statement of the following proposition:

Proposition 2.2: If $v(\quad)$ is concave then $\left\{\tilde{x}_{t}\right\}$ is mean reverting by Property III. ${ }^{5}$

Proof: See Exley et al. (2004).

If condition (4) were not satisfied, then increases in the value of the series would, on average, be followed by further increases and declines by further declines. Accordingly, the range of possible evolutions of a series would tend to fan out with the variance of the difference exploding as the difference in time indices grew, as in a random walk. Concavity of $v(\quad$ ) precludes this effect: increases in $\tilde{x}_{t}$ must be followed, on average, by eventual decreases and vice versa, a weak sense of mean reversion.

Property III is rarely employed in the empirical finance literature, and, as we demonstrate later, neither implies nor is it implied by either Property I or II. We will thus largely focus on Properties I and II since they are the most frequently cited.

$$
\text { IV. } \quad E_{t}\left[\tilde{x}_{t+1}-x_{t}\right]=\kappa\left[x_{t}-\bar{x}\right],
$$

where $E_{t}$ is the period $t$ conditional expectations operator, $\bar{x}$ is the unconditional mean of the series $\left\{\tilde{x}_{t}\right\}$ and $\kappa<0$ a constant. $^{6}$

\footnotetext{
${ }^{5}$ If $v(\quad)$ is convex then $\left\{\tilde{x}_{t}\right\}$ is mean averting

${ }^{6}$ This characterization is emphasized in Bekaert and Hodrick (2017). It is also expressed as $E_{t}\left[\ln \left(\tilde{x}_{t+1}\right)-\ell n\left(x_{t}\right)\right]=\kappa\left[\ln \left(x_{t}\right)-\bar{x}\right]$.
} 
Property IV identifies a mean reverting process as one that is always being "pulled towards its mean": if, at some $t$, the process is above its mean $\left(x_{t}>\bar{x}\right)$, the expected change next period should be negative and vice versa, with the "strength" of the pull-back determined by $\kappa$ and the extent of the current

deviation $\left[x_{t}-\bar{x}\right]$. Of all the characterizations of mean reversion this is the most intuitively obvious.

As noted in the introduction, the notion of mean reversion is one of aboveaverage realizations of the series being regularly followed by below-average values and vice versa. Which of the above properties is consistent with this intuition? Are the properties consistent with one another? To what extent do they refine the basic concept of stationarity? ${ }^{7}$ Since the context of these questions is one of equilibrium economic and financial time series, we choose to address them first within the framework of the simplest stochastic general equilibrium macroeconomic model.

\section{Modeling Perspective and the Baseline Paradigm}

We first focus on a simple representative agent neoclassical stochastic macroeconomic model with "planning" representation:

${ }^{7}$ As regards these questions, we can get an indication of the answer by looking at the simplest "canonical mean reverting process", an $\operatorname{AR}(1)$ :

$$
\tilde{x}_{t+1}=\rho x_{t}+\tilde{\varepsilon}_{t+1}, 0<\rho<1, \tilde{\varepsilon}_{t} \sim N\left(0, \sigma_{\tilde{\varepsilon}}^{2}\right) \text { for all } t
$$

For this process, $\operatorname{cov}\left(\tilde{x}_{t}, \tilde{x}_{t+1}\right)=\rho \sigma_{x_{t}}^{2}$ which is mean averting by Property I. Furthermore, since

$$
\operatorname{var}\left(\frac{\tilde{x}_{t}+\tilde{x}_{t+1}}{2}\right)=\frac{1+\rho}{1-\rho^{2}} \sigma_{\tilde{\varepsilon}}^{2}>\frac{\sigma_{\tilde{\varepsilon}}^{2}}{1-\rho^{2}}=\operatorname{var}\left(\tilde{x}_{t}\right)
$$

it is not mean reverting by Property II, confirming Proposition 2.1. As for Property III,

$$
\operatorname{cov}\left(\tilde{x}_{s}-\tilde{x}_{r}, \tilde{x}_{u}-\tilde{x}_{t}\right)=\operatorname{cov}\left(\sum_{j=r+1}^{s} \tilde{\varepsilon}_{j} \rho^{j-s}, \sum_{j=r+1}^{s} \tilde{\varepsilon}_{j} \rho^{u-s}\right)=0, \text { for all } r<s<t<u
$$

hence the process is also not Property III mean reverting. Lastly,

$$
E_{t}\left(\tilde{x}_{t+1}-x_{t}\right)=E_{t}\left((\rho-1) x_{t}+\tilde{\varepsilon}_{t+1}\right)=(\rho-1)\left(x_{t}-\bar{x}\right), \text { since } \bar{x}=0 .
$$

An AR(1) process is set up trivially to satisfy Property IV. 


$$
\begin{array}{ll}
\max & E\left(\sum_{t=0}^{\infty} \beta^{t} u\left(\tilde{c}_{t}, 1-\tilde{n}_{t}\right)\right) \\
\text { s.t. } & c_{t}+i_{t} \leq y_{t}=f\left(k_{t}, n_{t}\right) \tilde{\lambda}_{t} \\
& k_{t+1}=(1-\Omega) k_{t}+i_{t}, k_{0} \text { given, } 0 \leq n_{t} \leq 1 . \\
& \tilde{\lambda}_{t+1} \sim G\left(\tilde{\lambda}_{t+1} ; \lambda_{t}\right) .
\end{array}
$$

Adopting the customary notation, $u\left(c_{t}, 1-n_{t}\right)$ represents the representative agent's period utility function defined over his period $t$ consumption $c_{t}$ and leisure, $\left(1-n_{t}\right)$, where $n_{t}$ is labor supplied, $f\left(k_{t}, n_{t}\right) \lambda_{t}$ denotes the representative firm's CRS production function of capital stock $k_{t}$ and labor supplied with $\left\{\tilde{\lambda}_{t}\right\}$ the stochastic total factor productivity shock. The probability distribution function for $\left\{\tilde{\lambda}_{t+1}\right\}$ conditional on $\lambda_{t}$ is denoted $\mathrm{G}\left(\lambda_{t+1} ; \lambda_{t}\right)$ and is assumed to be known to the representative agent. ${ }^{8}$ Lastly, $\beta$ denotes the representative agent's subjective time discount factor and $\Omega$ the period depreciation rate.

As the notation suggests, the state variables for this economy are $k_{t}$ and $\lambda_{t}$. Under standard assumptions, problem (6) has a solution:

(i) continuous, time-invariant consumption $c_{t}=c\left(k_{t}, \lambda_{t}\right)$, investment $i_{t}=i\left(k_{t}, \lambda_{t}\right)$ and labor service $n_{t}=n\left(k_{t}, \lambda_{t}\right)$ functions exist that solve problem (1), and

(ii) a unique invariant probability measure on the state variable pair $\left(k_{t}, \lambda_{t}\right)$ exists to which the joint stochastic process on $\left(k_{t}, \lambda_{t}\right)$ converges weakly and which describes its long run behavior. With these attributes we say that the

\footnotetext{
${ }^{8}$ The productivity disturbance $\left\{\tilde{\lambda}_{t}\right\}$ will typically be of the form $\tilde{\lambda}_{t}=e^{\tilde{x}_{t}}$ where $\tilde{x}_{t}$ is an $\operatorname{AR}(1)$ process.
} 
joint process on $\left(k_{t}, \lambda_{t}\right)$ is stationary. ${ }^{9},{ }^{10}$ As a result the stochastic processes

governing investment, $i\left(k_{t}, \lambda_{t}\right)$, consumption, $\mathrm{c}\left(k_{t}, \lambda_{t}\right)$, labor service, $\mathrm{n}\left(k_{t}, \lambda_{t}\right)$ and output $y_{t}=y\left(k_{t}, n\left(k_{t}, \lambda_{t}\right)\right) \lambda_{t}$ are also stationary. The same investment and consumption functions arising as the solution to (6) coincide with the aggregate investment and consumption functions arising from an analogous decentralized market economy in recursive competitive equilibrium, a fact well known to the literature; see, e.g., Prescott and Mehra (1980), or Brock (1982).

These decentralization schemes for (6) may be generalized to accommodate an implied financial market where risk free debt and equity are competitively traded. ${ }^{11}$ Under this expanded interpretation, the period $t$ dividend satisfies

$$
d_{t}=f\left(k_{t}, n_{t}\right) \lambda_{t}-w_{t} n_{t}-i_{t}
$$

while the ex-dividend aggregate equity price, $p_{t}^{e}=p^{e}\left(k_{t}, \lambda_{t}\right)$, is identified with next period's capital stock:

\footnotetext{
${ }^{9}$ Our notion of (strong) stationarity for a discrete time Markov process $\{x\}$ is as follows: let $s, t$ be arbitrary time indices and $X$ the state space with $\hat{x} \in X$, and $B \subseteq X, B$ a subset.

Define $P(s, \hat{x}, t, B)=\operatorname{Prob}\left(x_{t} \in B ; x_{s}=\hat{x}\right)$. Then for any integer $u$, $P(s+u, \hat{x}, t+u, B)=\operatorname{Prob}\left(x_{s+u}=\hat{x} ; x_{t+u} \in B\right)=P(s, \hat{x}, t, B)$, the Markov process is said to be stationary. The same Markov process possesses an invariant distribution $\hat{G}($ ) on $X$ if and only if for any $B \subseteq X$,

$$
\left.\operatorname{Prob}\left(x_{t+1} \in B\right)=\int_{x \in X} P\left(x_{t+1} \in B ; x_{t}=x\right) \hat{G}(d x)\right) .
$$

All the stochastic processes analyzed in this article are Markov, stationary and possess unique invariant distributions defined on compact sets.

${ }^{10}$ The details behind these assertions can be found in the literature. Part (i) is entirely standard. As for part (ii), the stochastic kernel, the expression $P(s, \hat{x}, t, B)$ in footnote 3 can be shown to be increasing, order reversing and to satisfy the "Feller Property." By Theorem 3.2 in Kamihigashi and Stachurski (2014) a unique stationary probability distribution exists with the indicated properties.

${ }^{11}$ As such, the financial market can be regarded as "complete."
} 


$$
p_{t}^{e}=k_{t+1} \cdot{ }^{12}
$$

In (7) $w_{t}$ denotes the competitive wage rate, which, in equilibrium, satisfies

$$
w_{t}=f_{2}\left(k_{t}, n_{t}\right) \lambda_{t}
$$

Accordingly,

$$
1+r_{t+1}^{e}=\frac{p_{t+1}^{e}+d_{t+1}}{p_{t}^{e}}=f_{1}\left(k_{t+1}, n_{t+1}\right) \lambda_{t+1}+(1-\Omega) \quad \text { (by CRS) }
$$

where $r_{t+1}^{e}=f_{1}\left(k_{t+1}, n_{t+1}\right) \lambda_{t+1}-\Omega$ denotes the net return on unlevered equity from the "end of period t" to the "end of period $t+1 . " 13$

The period price, $p_{t}^{b}$, of a risk-free bond paying one unit of consumption in period $t+1$, irrespective of the realized state, is

$$
p_{t}^{b}=p^{b}\left(k_{t}, \lambda_{t}\right)=\beta \int \frac{u_{1}\left(\tilde{c}_{t+1}, 1-\tilde{n}_{t+1}\right)}{u_{1}\left(c_{t}, 1-n_{t}\right)} d G\left(\tilde{\lambda}_{t+1} ; \lambda_{t}\right)
$$

with the risk-free rate $r_{t}^{b}=r^{b}\left(k_{t-1}, \lambda_{t-1}\right)$ satisfying $\left(1+r_{t+1}^{b}\right)=1 / p_{t}^{b}$. Accordingly, the equity premium is defined by $r_{t}^{p}=r_{t}^{e}-r_{t}^{b}$.

As continuous bounded functions of the economy's state variables, $p^{e}\left(k_{t}, \lambda_{t}\right)$, $p^{b}\left(k_{t}, \lambda_{t}\right), r^{e}\left(k_{t}, \lambda_{t}\right), r^{b}\left(k_{t}, \lambda_{t}\right)$ and $r_{t}^{p}\left(k_{t}, \lambda_{t}\right)$ are also stationary stochastic processes.

\subsection{The Baseline Model}

We first restrict problem (6) by requiring that:

$$
u\left(c_{t}\right)=\ln \left(c_{t}\right), y_{t}=f\left(k_{t}, n_{t}\right) \tilde{\lambda}_{t}=\tilde{k}_{t}^{\alpha} \tilde{\lambda}_{t}, n_{t} \equiv 1, \Omega=1
$$

\footnotetext{
${ }^{12}$ In a related study, Lansing (2015) refers to this dividend expression as the "macroeconomic dividend." With this identification the dividend is assumed to be exclusively financed out of capital's income share.

${ }^{13}$ Identification (9) does not hold in more elaborate models with costs of adjusting the capital stock.
} 
and $\left\{\tilde{\lambda}_{t}\right\}$ a strictly positive i.i.d. stochastic process.

It is widely known that the optimal policy functions assume the form

$$
\begin{aligned}
& c_{t}=c\left(k_{t}, \lambda_{t}\right)=(1-\alpha \beta) y_{t} \text { and } \\
& i_{t}=k_{t+1}=\alpha \beta y_{t}=\alpha \beta k_{t}^{\alpha} \lambda_{t}
\end{aligned}
$$

Accordingly,

$$
\begin{aligned}
& p_{t}^{e}=p\left(k_{t}, \lambda_{t}\right)=k_{t+1}=\alpha \beta k_{t}^{\alpha} \lambda_{t}, \\
& p_{t}^{b}=p_{t}^{b}\left(k_{t}, \lambda_{t}\right)=\left(\beta E\left(\lambda_{t}^{-1}\right) /(\alpha \beta)^{\alpha}\right) k_{t}^{\alpha(1-\alpha)} \lambda_{t}^{1-\alpha} \\
& d_{t}=\alpha k_{t}^{\alpha} \lambda_{t}-\alpha \beta k_{t}^{\alpha} \lambda_{t}=\alpha(1-\beta) k_{t}^{\alpha} \lambda_{t} \cdot{ }^{14}
\end{aligned}
$$

With $\left\{\tilde{\lambda}_{t}\right\}$ an i.i.d. process, Hopenhayn and Prescott (1992) have shown that the derived stochastic process on capital stock is stationary and that there exists a corresponding ergodic probability distribution which captures its long run behavior; the same can thus be said for $p_{t}^{e}, p_{t}^{b}$ and $d_{t}$.

We first explore this model with reference to Property I.

Proposition 3.1: In model (6) specialized as per identifications (11) and (12), the equity price $\left\{\tilde{p}_{t}^{e}\right\}$, the default-free bond price $\left\{\tilde{p}_{t}^{b}\right\}$ and the dividend series $\left\{\tilde{d}_{t}\right\}$ are all mean averting by Property $\mathrm{I}^{15}$

Proof: See the Technical Appendix.

This result confirms that the notions of Property I mean reversion and stationarity (the existence of a long run ergodic probability distribution on capital stock - the equity price - to which the economy converges) are not

${ }^{14}$ By recursive substitution $k_{t}=\left[(\alpha \beta)^{1+\alpha+\alpha^{2}+\ldots \alpha^{t-1}}\right] k_{0}^{\alpha^{t}} \prod_{s=0}^{t-1} \lambda_{s}^{\alpha^{t-1-s}}$.

${ }^{15}$ The part of Proposition 3.1 dealing specifically with $\left\{\tilde{p}_{t}^{e}\right\}$ was first presented in Basu and Vinod (1994) and Basu and Samanta (2001) in a slightly less general setting. We extend their explorations with a different goal in mind. 
equivalent, and that the distinction arises in the simplest equilibrium macroeconomic models.

Corollary 3.1 Dividends, equity prices and default-free debt prices are mean averting under Property II.

Proof: A consequence of Proposition 2.1 (see footnote 5).

Furthermore, these observations are generic in the sense expressed in the following result:

Proposition 3.2: Consider any equilibrium model of the general form (6) for which the equilibrium investment function $i\left(k_{t}, \lambda_{t}\right)$ is continuous and increasing in both its arguments. Suppose also that the period $t$ price of equity and the period $t+1$ level of the capital stock coincide (no costs of adjustment). Then under both Properties I and II $\left\{\tilde{p}_{t}^{e}\right\}$ will be mean averting. If $p_{t}^{b}=h\left(k_{t}, \lambda_{t}\right)$, where $h(\cdot)$ is continuous and increasing in both its arguments, then $\left\{\tilde{p}_{t}^{b}\right\}$ will be mean averting as well.

Proof: See the Technical Appendix.

Most of the macroeconomic models to be considered in this paper satisfy the conditions of the above proposition. Hence the results in Proposition 3.2 apply quite generally.

We next examine mean reversion (Property I) in the equity and bond return series for this model.

Proposition 3.3: For Model (6), specialized by (11) and (12):

a. $\operatorname{corr}\left(\tilde{r}_{t}^{e}, \tilde{r}_{t+1}^{e}\right) \geq-M, M>0$; so that equity returns may be mean reverting by Property I.

b. $\operatorname{corr}\left(\tilde{r}_{t}^{b}, \tilde{r}_{t+1}^{b}\right)>0$; i.e., bond returns are mean averting by Property I.

Proof: See the Technical Appendix. 
It is clear (see the Proof of Proposition 3.3) that the concavity of the production function $(\alpha-1)<0$ plays the key role in inducing mean reversion (as characterized by Property I) in equity returns, a fact first observed in Basu and Vinod (1994). Risk-free returns, however, are mean averting.

Taken together, Propositions 3.1 and 3.3 remind us that mean reversion in equity returns need not imply mean reversion in equity prices (at least by the criterion of Property I). ${ }^{16}$ That mean reversion in returns is compatible with mean aversion in prices is already well known (Spierdijk and Bikker (2012)). It is surprising, however, to find this compatibility in the simplest possible dynamic equilibrium context.

It is difficult to derive fully general results for Property III without further specialization of the productivity process. ${ }^{17}$ Consider Proposition 3.4.

Proposition 3.4: Consider model (6) specialized as per (11) with a productivity shock of the form $\left\{e^{\tilde{\lambda}_{t}}\right\}$, where $\tilde{\lambda}_{t}=\tilde{\varepsilon}_{t},\left\{\tilde{\varepsilon}_{t}\right\}$ i.i.d. $N\left(0, \sigma_{\varepsilon}^{2}\right)^{18}$. Then,

(i) The equity price series and dividend series are mean averting by Property III;

(ii) The return on equity is mean reverting by Property III.

Proof: See Donaldson et al. (2015).

The proof of Proposition 3.4 reveals that concavity in production $(\alpha<1)$ is also key in generating Property III mean reversion in equity returns. These results are entirely consistent with those obtained for our earlier analysis of Properties I and II. Nevertheless, the fact that Property III is not discriminating

\footnotetext{
${ }^{16}$ Indeed, in this simple model both equity prices and dividends are mean averting, yet equity returns are nevertheless mean reverting.

${ }^{17}$ To show, for example, that $\left\{\tilde{p}_{t}^{e}\right\}$ is mean averting, it is sufficient to show $\operatorname{var}\left\{\tilde{p}_{t}^{e}-\tilde{p}_{t}^{s}\right\}$ is convex as a function of $t$-s, where $\tilde{p}_{t}^{e}=\tilde{k}_{t}=\left[(\alpha \beta)^{1+\alpha+\alpha^{2}+\ldots+\alpha^{t-1}}\right] k_{0}^{\alpha^{t}} \prod_{j=0}^{t-1} \tilde{\lambda}_{j}^{\alpha^{t-1-j}}$. Variances of products of random variables are complex quantities.

${ }^{18}$ This shock process is typically used in the business-cycle-literature.
} 
across $\mathrm{AR}(1)$ processes for various $\rho$ tends to disqualify it as a critical mean reversion characterization.

For the Baseline Model, Property IV is not satisfied; there appears no single $\kappa$ for which condition (5) holds. It is strongly mean reverting, however, in the sense that if $\tilde{\varepsilon}_{t} \equiv 0$ for all $t$, then $k_{t}=p_{t}^{e}<\bar{p}_{t}^{e}=\bar{k}$ implies $p_{t+1}^{e}>p_{t}^{e}$ and vice versa if $p_{t}^{e}>\bar{p}_{t}^{e}$.

If the notion of mean reversion is intended to capture the property that above average values of a stochastic process must regularly be followed by below average values, then all the series considered thus far, $\left\{\tilde{p}_{t}^{e}\right\},\left\{\tilde{d}_{t}\right\},\left\{\tilde{r}_{t}^{e}\right\},\left\{\tilde{p}_{t}^{b}\right\}$, and

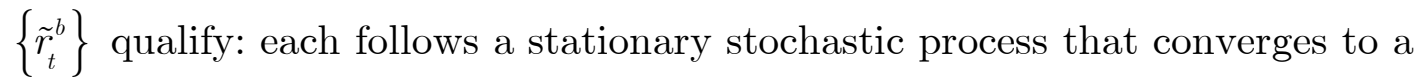
unique, irreducible ergodic set. Yet, as Propositions 3.1. - 3.3 make clear, (i) mean aversion in a time series (by Properties I - III) does not imply nonstationary, and (ii) stationary of a series does not guarantee mean reversion by any of the Properties I - IV.

Properties I - IV thus appear to represent artificially restrictive distinctions relative to the basic intuitive sense of a mean reverting series. A more useful characterization of mean reversion should allow easy comparisons of the following sort: when is one time series more highly mean-reverting than another? Intuitively, a more highly mean reverting series should cross its mean more often; that is, with greater "frequency." ${ }^{19}$ Crossing the mean with greater frequency must in turn imply less persistence as regards the series being exclusively either in states above or below its mean. In the next section we develop this concept of persistence and relate it to the various characterizations of mean reversion presented thus far.

${ }^{19}$ A crossing of the mean from above at time $t$ would signify that $x_{t} \geq \bar{x}$, yet $x_{t+1}<\bar{x}$, and analogously for crossing the mean from below. 


\section{An Alternative Metric}

In view of the preceding discussion, we propose "Average Crossing Time" $(A C T)$ as a simple measure of persistence. Intuitively a larger $A C T$ would roughly correspond to less frequent crossings of the mean in turn suggesting weaker mean reversion. We offer the simple $A C T$ measure because not only does it help to evaluate Properties I - IV, but because it that provides an intuitive sense of one series being more weakly mean reverting than another.

A discrete-time stochastic process's average crossing time $(A C T)$ is the average number of time periods before the process transitions from above its unconditional mean to below its unconditional mean or vice versa. Subject to certain modest refinements, the $A C T$ can be computed by dividing the length of the series by the number of crossings of the mean observed over its duration. Under this concept, an economic time series is said to be mean reverting if and only if its $A C T$ is finite. To gain some intuition for the $A C T$-autocorrelation relationship, we computed these quantities for the financial time series generated by the Baseline model. Table 4.1 presents the results.

Table 4.1

$$
\begin{aligned}
& \text { Baseline Model: Correlations and } A C T \mathrm{~s}^{(\mathrm{i})} \\
& \alpha=.36, \beta=.99, \Omega=1,\left\{\tilde{\lambda}_{t}\right\} \text { i.i.d., } \sigma_{e}^{2}=.00712 \\
& \operatorname{corr}\left(\tilde{p}_{t}^{e}, \tilde{p}_{t+1}^{e}\right) \quad \operatorname{corr}\left(\tilde{p}_{t}^{b}, \tilde{p}_{t+1}^{b}\right) \quad \operatorname{corr}\left(\tilde{r}_{t}^{e}, \tilde{r}_{t+1}^{e}\right) \quad \operatorname{corr}\left(\tilde{r}_{t}^{b}, \tilde{r}_{t+1}^{b}\right) \quad \operatorname{corr}\left(\tilde{r}_{t}^{p}, \tilde{r}_{t+1}^{p}\right) \\
& .39 \\
& .39 \\
& -.30 \\
& .39 \\
& .03 \\
& A C T\left(\tilde{p}_{t}^{e}\right) \\
& A C T\left(\tilde{p}_{t}^{b}\right) \\
& A C T\left(\tilde{r}_{t}^{e}\right) \\
& A C T\left(\tilde{r}_{t}^{b}\right) \\
& A C T\left(\tilde{r}_{t}^{p}\right) \\
& 1.66 \\
& 2.70
\end{aligned}
$$

Two observations stand out. First, the ordering (smallest to largest) of correlations and $A C T \mathrm{~s}$ is the same, subject to rounding and numerical approximations: a more positive autocorrelation is associated with a larger $A C T$, 
which implies less frequent "crossings." Second, the single negatively autocorrelated series, $\left\{r_{t}^{e}\right\}$, is the only one for which the $A C T$ is less than two. In the remainder of this section, we explore the generality of these observations.

First note that the state variables for any DSGE model follow a Markov process for an appropriately defined state space and thus can be wellapproximated by a Markov chain of sufficiently high dimension. The same is true for all the endogenous return series arising in equilibrium. Accordingly, we focus our attention on chain representations. Furthermore, when appropriately constructed, a specific two state Markov chains turns out to be all that is necessary for the $A C T$ computation.

To see this, consider an $N$ state irreducible Markov chain $\left\{\tilde{\gamma}_{t}\right\}$ with states indexed by $\gamma_{i}, \gamma_{j}, i, j: 1,2, \ldots, N$, transition probabilities $\phi_{i j}, i=1,2, \ldots, N, j=1,2, \ldots, N$ and ergodic probabilities $\pi_{i}: i=1,2, \ldots, N, \pi_{i}>0 \forall i .{ }^{20}$ We will subsequently interpret these quantities as return or price measurements. Let T denote the chain's transition probability matrix with entries $\phi_{i j}$. Without much loss of generality, we may assume there exists a state $\hat{j}$ such that

$$
\gamma_{\hat{j}+1}<E\left(\tilde{\gamma}_{t}\right)<\gamma_{\hat{j}}
$$

a restriction that allows the unambiguous definition of the sets $\gamma^{A}$ and $\gamma^{B}$, where

$$
\gamma^{A}=\left\{\gamma_{j}: \gamma_{j}>E\left(\tilde{\gamma}_{t}\right)\right\}, \gamma^{B}=\left\{\gamma_{j}: \gamma_{j}<E\left(\tilde{\gamma}_{t}\right)\right\} \text { and } \gamma^{A} \cap \gamma^{B}=\varnothing .^{21}
$$

We construct a derivative (two state) Markov chain $\left\{\tilde{\gamma}_{t}^{A B}\right\}$ on the sets $\gamma^{A}$ and $\gamma^{B}$ by defining its $\gamma^{A}, \gamma^{B}$ transition probabilities as follows:

${ }^{20}\left\{\tilde{\gamma}_{t}\right\}$ will subsequently represent equilibrium price or return series.

${ }^{21}$ If, for some $j^{*}, \gamma_{j^{*}}=E\left(\tilde{\gamma}_{t}\right)$, then $\gamma_{j^{*}}$ is in neither $\gamma^{A}$ nor $\gamma^{B}$ which distorts the relationship of the original transition matrix, which includes $\gamma_{j^{*}}$, to the "aggregated" process $\left\{\tilde{\gamma}_{t}^{A B}\right\}$. 


$$
\begin{aligned}
& \phi_{A A}=\operatorname{Prob}\left(\tilde{\gamma}_{t+1} \in \gamma^{A} \mid \gamma_{t} \in \gamma^{A}\right)=\sum_{j=1}^{\hat{j}}\left(\left(\frac{\pi_{j}}{\sum_{\ell=1}^{\hat{j}} \pi_{\ell}}\right)\left(\sum_{k=1}^{\hat{j}} \phi_{j k}\right)\right), \\
& \phi_{A B}=\operatorname{Prob}\left(\tilde{\gamma}_{t+1} \in \gamma^{B} \mid \gamma_{t} \in \gamma^{A}\right)=\sum_{j=1}^{\hat{j}}\left(\left(\frac{\pi_{j}}{\sum_{\ell=1}^{\hat{j}} \pi_{\ell}}\right)\left(\sum_{k=\hat{j}+1}^{N} \phi_{j k}\right)\right), \\
& \phi_{B A}=\operatorname{Prob}\left(\tilde{\gamma}_{t+1} \in \gamma^{A} \mid \gamma_{t} \in \gamma^{B}\right)=\sum_{j=\hat{j}+1}^{N}\left(\left(\frac{\pi_{j}}{\sum_{\ell=\hat{j}+1}^{N} \pi_{\ell}}\right)\left(\left(\sum_{k=1}^{\hat{j}} \phi_{j k}\right)\right)\right. \text { and } \\
& \phi_{B B}=\operatorname{Prob}\left(\tilde{\gamma}_{t+1} \in \gamma^{B} \mid \gamma_{t} \in \gamma^{B}\right)=\sum_{j=\hat{j}+1}^{N}\left(\left(\frac{\sum_{\ell=\hat{j}+1}^{N} \pi_{\ell}}{\pi_{j}}\right)\left(\sum_{k=\hat{j}+1}^{N} \phi_{j k}\right)\right) .
\end{aligned}
$$

Let $T^{A B}$ denote the transition probability matrix with the above entries,

$$
T^{A B}=\gamma^{A}\left[\begin{array}{ll}
\gamma^{A} & \gamma^{B} \\
\phi_{A A} & \phi_{A B} \\
\phi_{B A} & \phi_{B B}
\end{array}\right],
$$

and denote the long run ergodic probabilities governing the relative frequency of observing, respectively, elements of $\gamma^{A}$ and $\gamma^{B}$ by $\pi_{A}$ and $\pi_{B}$ where

$$
\pi_{A}=\sum_{\gamma_{j} \in \gamma^{A}} \pi_{j}, \text { and } \pi_{B}=\sum_{\gamma_{j} \in \gamma^{B}} \pi_{j} .
$$

For any discrete time Markov process $\left\{\tilde{\eta}_{t}\right\}$, we define the average crossing time from above as the average number of periods the process assumes values above its mean, inclusive of the first period it "crosses"; i.e., assumes a value 
below its mean. We denote this quantity by $A C T_{\left\{\tilde{n}_{t}\right\}}^{A}$. The average crossing time from below is defined analogously and is denoted by $A C T_{\left\{\tilde{\eta}_{t}\right\}}^{B}$. In the case of the two state Markov chain $\left\{\tilde{\gamma}_{t}^{A B}\right\}$, these quantities are easily computed as follows:

$$
\begin{aligned}
A C T_{\left\{\tilde{\gamma}_{t}^{A B}\right\}}^{A} & =\sum_{n=1}^{\infty} n \operatorname{Prob}\left(\tilde{\gamma}_{t+n}^{A B} \in \gamma^{B} \mid \gamma_{t+j}^{A B} \in \gamma^{A}, j=0,1,2, \ldots, n-1\right) \\
& =\sum_{n=1}^{\infty} n\left(\phi^{A A}\right)^{n-1}\left(1-\phi^{A A}\right) \\
& =\frac{\left(1-\phi^{A A}\right)}{\phi^{A A}} \sum_{n=1}^{\infty} n\left(\phi^{A A}\right)^{n}=\frac{1-\phi^{A A}}{\phi^{A A}} \cdot \frac{\phi^{A A}}{\left(1-\phi^{A A}\right)^{2}}=\frac{1}{1-\phi^{A A}} .^{22}
\end{aligned}
$$

Similarly,

$$
A C T_{\left\{\tilde{\gamma}_{t}^{A B}\right\}}^{B}=\sum_{n=1}^{\infty} n \operatorname{Prob}\left(\tilde{\gamma}_{t+n}^{A B} \in \gamma^{A} \mid \gamma_{t+j}^{A B} \in \gamma^{B}, j=0,1,2, \ldots, n-1\right)=\frac{1}{1-\phi^{B B}} .
$$

With these quantities in mind, we focus exclusively on the "set chain" $\left\{\tilde{\gamma}_{t}^{A B}\right\}$ rather than its antecedent, $\left\{\tilde{\gamma}_{t}\right\}$. The justification for this choice takes the form of a small proposition:

Proposition 4.1: For any irreducible Markov chain $\left\{\tilde{\gamma}_{t}\right\}$

$$
\begin{aligned}
& A C T_{\left\{\tilde{\gamma}_{t}\right\}}^{A}=A C T_{\left\{\tilde{\gamma}_{t}^{A B}\right\}}^{A} \text { and } A C T_{\left\{\tilde{\gamma}_{t}\right\}}^{B}=A C T_{\left\{\tilde{\gamma}_{t}^{A B}\right\}}^{B} . \text { Accordingly, } \\
& A C T_{\left\{\tilde{\gamma}_{t}\right\}}=A C T_{\left\{\tilde{\gamma}_{t}^{A B}\right\}}
\end{aligned}
$$

Proof: See the Technical Appendix.

${ }^{22}$ One could also define the average crossing time from $\gamma^{A}$ to $\gamma^{B}$ by the average time the process remains in state $\gamma^{A}$, not including the period of crossing. Identify this quantity as $\widehat{A C T}^{A}$ where $\widehat{A C T}^{A}=\sum_{n=1}^{\infty} n \operatorname{Prob}\left(\tilde{\gamma}_{t+n}^{A B} \in \gamma^{A} \mid \tilde{\gamma}_{t+n+1}^{A B} \in \gamma^{B}\right.$ and $\left.\gamma_{t+j}^{A B} \in \gamma^{A}, j=0,1,2, \ldots, n-1\right)=\frac{\phi^{A A}}{\left(1-\phi^{A A}\right)}$

Clearly $A C T^{A}=\widehat{A C T}^{A}+1$, as the respective formulae confirm. We choose to work with $A C T^{A}$ rather than $\widehat{A C T}^{A}$ and the analogous $A C T^{B}$ rather than $\widehat{A C T}^{B}$ as to do so proves to be algebraically simpler. 
Proposition 4.1 simply claims that the $A C T^{A}$ and $A C T^{B}$ values for the original chain and its derived "set chain" are identical. Hence, we shift our focus to the latter. We do not claim, however, that $\operatorname{corr}\left(\tilde{\gamma}_{t}, \tilde{\gamma}_{t+1}\right)=\operatorname{corr}\left(\tilde{\gamma}_{t}^{A B}, \tilde{\gamma}_{t+1}^{A B}\right)$, or that any other statistical properties beyond $A C T^{A}$ and $A C T^{B}$ are the same for both series.

We next initiate a straightforward characterization of $\left\{\tilde{\gamma}_{t}^{A B}\right\}$. Its properties are listed below. All the calculations are entirely straightforward and are provided in the Technical Appendix.
A. $\pi^{A}=\frac{1-\phi^{A A}}{2-\left(\phi^{A A}+\phi^{B B}\right)}$ and $\pi^{B}=\frac{1-\phi^{B B}}{2-\left(\phi^{A A}+\phi^{B B}\right)}$,

where $\pi^{A}, \pi^{B}$ represent, respectively, the ergodic probabilities of the process being in set $\gamma^{A}$ or $\gamma^{B}$.

B. As noted earlier, $A C T^{A}=\frac{1}{1-\phi^{A A}}$ and $A C T^{B}=\frac{1}{1-\phi^{B B}}$. Accordingly, "Average Crossing Time," ACT, satisfies

$$
\begin{aligned}
& \begin{aligned}
A C T_{\left\{\tilde{f}_{t}^{A B}\right\}} & =\pi^{A} A C T_{\left\{\tilde{\gamma}_{t}^{A B}\right\}}^{A}+\pi^{B} A C T_{\left\{\hat{\gamma}_{t}^{A B}\right\}}^{B} \\
& =\frac{1}{2-\left(\phi^{A A}+\phi^{B B}\right)}\left(\frac{1-\phi^{A A}}{1-\phi^{B B}}+\frac{1-\phi^{B B}}{1-\phi^{A A}}\right)
\end{aligned} \\
& \text { C. } \operatorname{corr}\left(\tilde{\gamma}_{t}^{A B}, \tilde{\gamma}_{t+1}^{A B}\right)=\left(\phi^{A A}+\phi^{B B}\right)-1 .
\end{aligned}
$$

From equation (19), we see that the same autocorrelation can arise from many different $\phi^{A A}, \phi^{B B}$ pairs; to illustrate, any $\left(\phi^{A A}, \phi^{B B}\right) \in\{(.5, .5),(.7, .3),(.05, .95)\}$ yields a $\operatorname{corr}\left(\tilde{\gamma}_{t}^{A B}, \tilde{\gamma}_{t+1}^{A B}\right)=0$, yet the corresponding $A C T$ s are, respectively, 2, 2.762, and 19.053. Independence, as measured by $\operatorname{corr}\left(\phi_{t}^{A B}, \phi_{t+1}^{A B}\right)=0$, can, in fact, be consistent with many patterns (as captured by the $A C T^{A}, A C T^{B}$ values). 
D. Proposition 4.2: If $A C T_{\left\{\tilde{\gamma}_{t}^{A B\}}\right.} \leq 2$, then $\operatorname{corr}\left(\tilde{\gamma}_{t}^{A B}, \tilde{\gamma}_{t+1}^{A B}\right) \leq 0$.

If $\phi^{A A}=\phi^{B B}$, then $\operatorname{corr}\left(\tilde{\gamma}_{t}^{A B}, \tilde{\gamma}_{t+1}^{A B}\right) \leq 0$ implies $A C T \leq 2$.

First observe that Proposition 4.2 is consistent with the values presented in Table 4.1: the only negative autocorrelation is $\operatorname{corr}\left(\tilde{r}_{t}^{e}, \tilde{r}_{t+1}^{e}\right)=-.30$ and its $A C T=1.66$, suggesting very frequent crossings. Note that the values in Table 4.1 also confirm the observation formalized as Proposition 4.1: although the statistics computed there are based on the full series " $\left\{\tilde{\gamma}_{t}\right\}$ " the ACT correlation relationship expressed in Proposition 4.2 for the " $\left\{\tilde{\gamma}_{t}^{A B}\right\}$ " series is observed.

As modest as it is, Proposition 4.2 is a result of interest. Essentially it says that if Properties I or II are to be satisfied, the process in question must have an $A C T$ of two or less which suggests very frequent crossings relative to the model time period. If real world data were subject to either Property I or II criteria, it is almost certain that tests for mean reversion will fail. To put it more starkly, the $A C T$ of "white noise" is also 2.

Figure 1 jointly illustrates observations $\mathrm{C}$ and $\mathrm{D}$ for various magnifications: in particular the range of $A C T \mathrm{~s}$ associated with any degree of autocorrelation, and that all $A C T \mathrm{~s} \leq 2$ are identified with negative autocorrelation. 


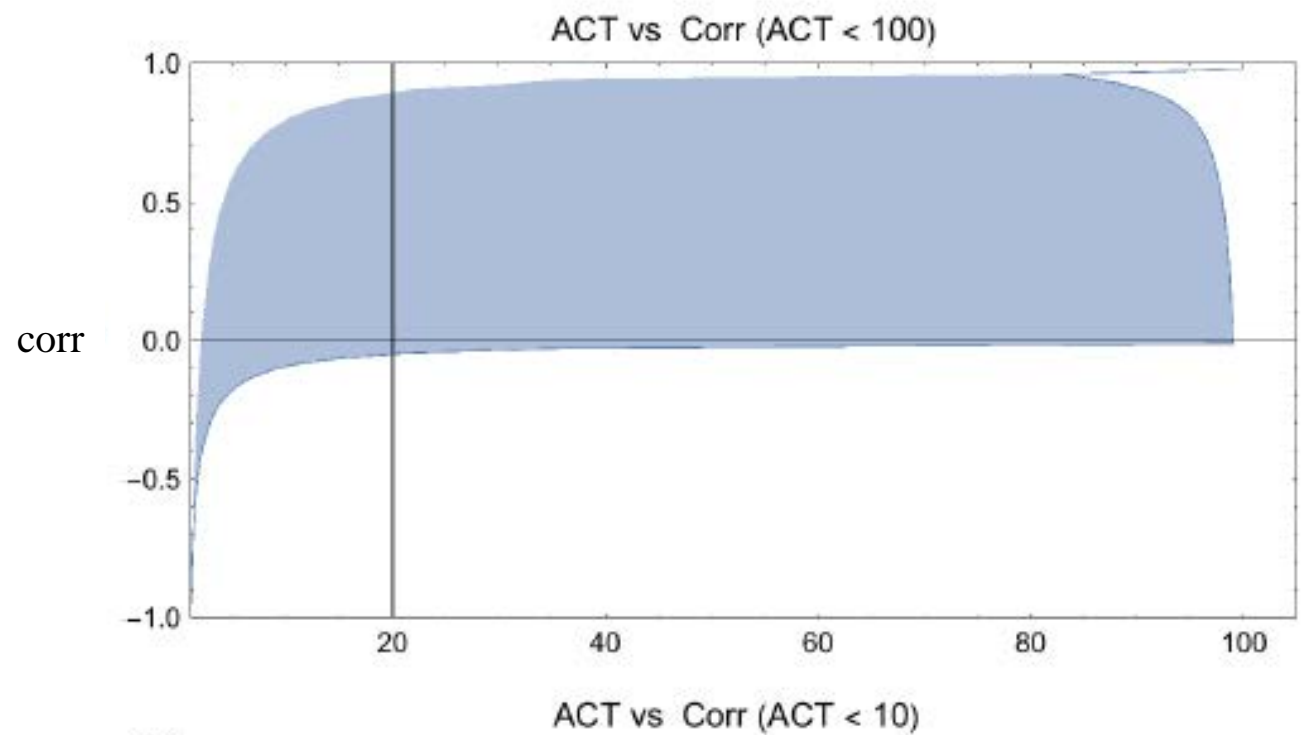

ACT

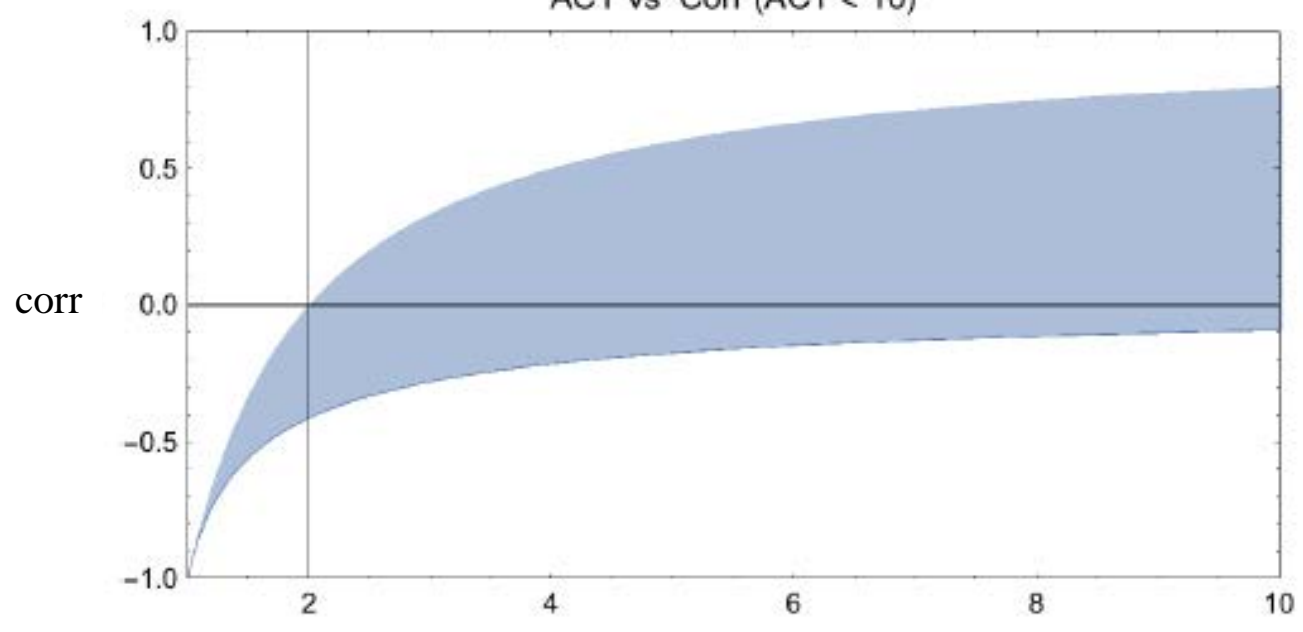

$A C T$

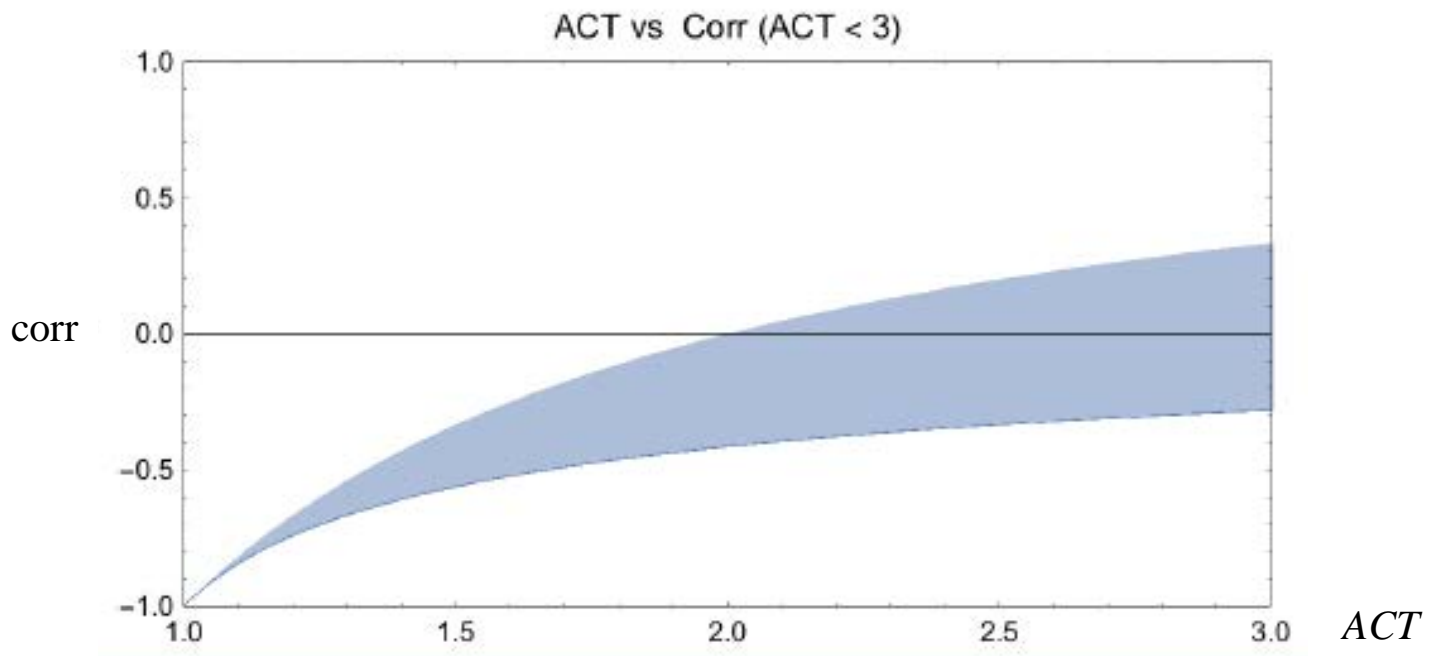

Figure 1. $A C T$ vs. Correlation 
E. Consider two distinct, irreducible two-state Markov chains, $\left\{\tilde{\gamma}^{x}\right\}$ and $\left\{\tilde{\gamma}^{y}\right\}$ with transition probability matrices $T^{x}$ and $T^{y}$, respectively, where

$$
T^{x}:=\begin{aligned}
& \gamma_{1}^{x} \\
& \gamma_{2}^{x}
\end{aligned}\left[\begin{array}{cc}
\phi_{1}^{x} & 1-\overline{\phi_{1}^{x}} \\
1-\phi_{2}^{x} & \phi_{2}^{x}
\end{array}\right] \quad \text { and } \quad T^{y}:=\begin{aligned}
& \gamma_{1}^{y} \\
& \gamma_{2}^{y}
\end{aligned}\left[\begin{array}{cc}
\phi_{1}^{y} & 1-\phi_{1}^{y} \\
1-\phi_{2}^{y} & \phi_{2}^{y}
\end{array}\right]
$$

Suppose $\phi_{1}^{x}=\phi_{2}^{x}=\phi^{x}$ and $\phi_{1}^{y}=\phi_{2}^{y}=\phi^{y}$. Then $\operatorname{corr}\left(\tilde{\gamma}_{t}^{x}, \tilde{\gamma}_{t+1}^{x}\right)>\operatorname{corr}\left(\tilde{\gamma}_{t}^{y}, \tilde{\gamma}_{t+1}^{y}\right)$

if and only if $A C T_{\left\{\tilde{\gamma}_{t}^{x}\right\}}>A C T_{\left\{\tilde{r}_{t}^{y}\right\}}$.

From the example in part (C) above, it is clear that the statement of our original motivating assertion is not generally true: a higher $A C T$ value is not equivalent to higher autocorrelation. Yet, this assertion appears to capture the relative autocorrelation- $A C T$ pattern observed in Table 4.1, which suggests that the underlying multi-state probability transition matrices are "approximately" symmetric, a fact that follows intuitively from the technology shock symmetry.

F. By continuity, assertion (E) can be generalized in the following way. Consider a family of irreducible, two state Markov chains of the form $\left\{\tilde{\gamma}_{t}^{A B}\right\}$. Let $\left\{\widehat{\tilde{\gamma}_{t}}\right\}$ and $\left\{\overline{\tilde{\gamma}_{t}}\right\}$ be two such chains and let us associate them with transition probability pairs $\left(\hat{\phi}^{A A}, \hat{\phi}^{B B}\right)$ and $\left(\bar{\phi}^{A A}, \bar{\phi}^{B B}\right)$, respectively. We are interested to identify the set $\mathrm{A}$, where

$$
\begin{aligned}
& A=\left\{\left(\bar{\phi}^{A A}, \bar{\phi}^{B B}\right) \text { : for any }\left(\hat{\phi}^{A A}, \hat{\phi}^{B B}\right) \text {, where (i) } \bar{\phi}^{A A} \leq \hat{\phi}^{A A}\right. \text { and (ii) } \\
& \left.\bar{\phi}^{B B} \leq \hat{\phi}^{B B} \text {, then (iii) } A C T_{\left\{\bar{\gamma}_{t}\right\}} \leq A C T_{\left\{\hat{\hat{t}}_{t}\right\}}\right\} .
\end{aligned}
$$

By $(\mathrm{C})$ it is also true that for set $A$ : (iv) $\operatorname{corr}\left(\overline{\tilde{\gamma}}_{t}, \bar{\gamma}_{t+1}\right) \leq \operatorname{corr}\left(\hat{\tilde{\gamma}}_{t}, \hat{\tilde{\gamma}}_{t+1}\right)$. If either inequality (i) or (ii) is strict, then (iii) and (iv) are strict. 
That the set $A$ is non-empty follows from $\mathrm{E}$ and continuity: there must exist a region surrounding the $45^{\circ}$ line (where $\phi^{A A}=\phi^{B B}$ ) for which greater $A C T \mathrm{~s}$ and greater (more positive) autocorrelations increase hand in hand. The question remains only "how large" the region is. The answer is: quite large. This region is portrayed in Figure 2 and was numerically constructed using the following alternative representation of the set A:

$$
A=\left\{\left(\phi^{A A}, \phi^{B B}\right): \partial A C T / \partial \phi^{A A} \geq 0 \text { and } \partial A C T / \partial \phi^{B B} \geq 0\right\} .
$$




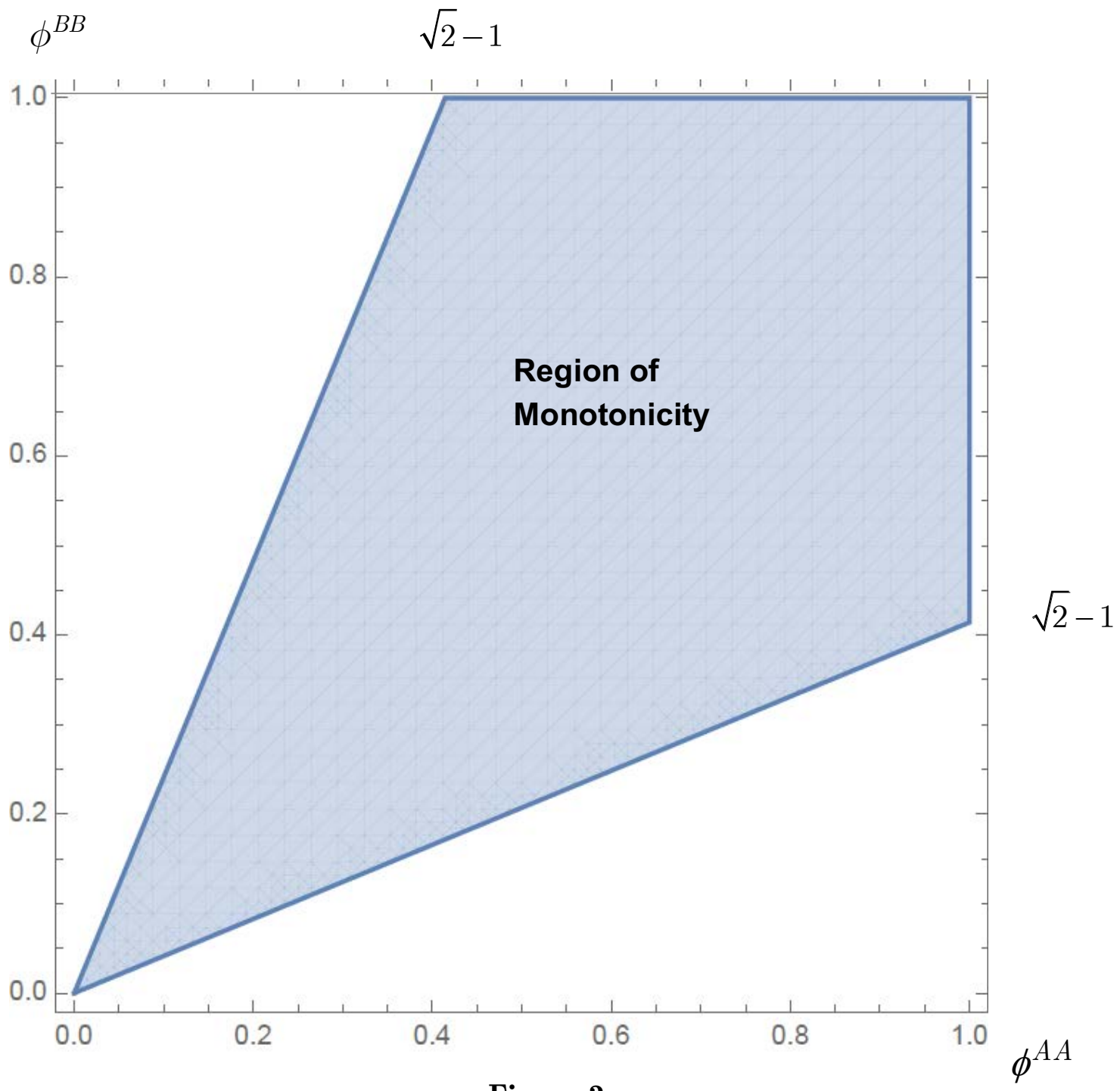

Figure 2

Transition Matrices for which Greater Autocorrelation Implies a Larger $A C T$ Value 
For $\left\{\tilde{\gamma}^{A B}\right\}$ that are far from symmetric (that is, $\phi^{A A}$ and $\phi^{B B}$ are very different; i.e., the non-shaded region of Figure 2), we do not observe $A C T$ and autocorrelation increasing in tandem as our original intuition suggested. While individually $\partial A C T^{A} / \partial \phi^{A A}>0$ and $\partial A C T^{B} / \partial \phi^{B B}>0$ uniformly, it does not necessarily follow that, e.g., $\partial A C T / \partial \phi^{A A}>0$. To illustrate, compare the $A C T$ s for $\phi^{A A}, \phi^{B B}=(.9, .05)$ and $\phi^{A A}, \phi^{B B}=(.9, .10)$ :

$$
\begin{aligned}
& A C T_{\left(\phi^{A A}=.9, \phi^{B B}=.05\right)}=9.148 \\
& A C T_{\left(\phi^{A A}=.9, \phi^{B B}=.10\right)}=9.111
\end{aligned}
$$

The phenomenon arises because the relative stationary probabilities change as $\phi^{B B}$ increases: more probability weight is placed on the lower $A C T^{B B}<A C T^{A A}$, causing the overall $A C T$ to decline. If $\phi^{A A} \approx \phi^{B B}$, there is little change in the corresponding $\left(\pi^{A}, \pi^{B}\right)$ when either $\phi^{A A}$ or $\phi^{B B}$ is marginally increased, so that the more intuitive relationship between the $A C T$ and its corresponding autocorrelation is observed.

Observation $\mathrm{C}$ also suggests that to identify mean reversion solely with either Property I or II is to forgo information. Any value that this particular measurement assumes is clearly compatible with a wide range of stochastic structures $\left(\phi^{A A}, \phi^{B B}\right)$. It is rather the knowledge of $A C T^{A}$ and $A C T^{B}$ that is critical to a comprehensive description of a "mean-reverting" process for investors. If the intuitive notion of "mean reversion" is the sense of "frequent crossings," then as regards the $A C T$ measurement, a mean reverting process by Properties I or II is not far from a sequentially independent one.

The results in Table 4.1 are also in the spirit of Observations E and F. Recall that the time series on which the values reported in Table 4.1 are generated arise by approximating the underlying real economy about its steady state. Given i.i.d. productivity shocks, the economy evolves symmetrically (about 
its steady state), suggesting that to a first approximation $\phi^{A A} \approx \phi^{B B}$. The lockstep increases in $A C T$ and correlation that are manifest in Table 4.1 follow naturally from Observation F; equivalently, the series described in Table 4.1 all have corresponding $\left(\phi^{A A}, \phi^{B B}\right) \in A$.

Note also that the statistics computed for Table 4.1 were created from the basic underlying $\left\{\tilde{\gamma}_{t}\right\}$ processes rather than its "aggregate state" process $\left\{\tilde{\gamma}_{t}^{A B}\right\}$. The fact that the relationships of Proposition 4.2, which are based on the transformed $\left\{\tilde{\gamma}_{t}^{A B}\right\}$, are borne out in $A C T$ data generated by the original $\left\{\tilde{\gamma}_{t}\right\}$ again confirms that $A C T\left(\tilde{\gamma}_{t}^{A B}\right)=A C T\left(\tilde{\gamma}_{t}\right)$ as per Proposition 4.1.

Certain distinctive results in Table 4.1 are unique to the Baseline parameterization (in particular, to the $\rho=0, \Omega=1$ assumptions). In particular $\operatorname{corr}\left(\tilde{p}_{t}^{e}, \tilde{p}_{t+1}^{e}\right)=\operatorname{corr}\left(\tilde{p}_{t}^{b}, \tilde{p}_{t+1}^{b}\right)$ and $A C T\left(\tilde{p}_{t}^{e}\right)=A C T\left(\tilde{p}_{t}^{b}\right)$. These identities follow from the fact that $p_{t}^{b}=\frac{E\left(\lambda_{t}^{-1}\right)}{\alpha}\left(p_{t}^{e}\right)^{1-\alpha}$. Accordingly, $\left\{\tilde{p}_{t}^{b}\right\}$ exceeds its mean when $\left\{p_{t}^{e}\right\}$ does and vice versa. With identical $A C T \mathrm{~s}$, their autocorrelations must be identical. Furthermore, since $r_{t}^{b}=\frac{1}{p_{t}^{b}}-1, r_{t}^{b}$ will exceed its mean if and only if $p_{t}^{b}$ falls short of its mean, and vice versa, leading to identical $A C T$ s for $\left\{\tilde{p}_{t}^{b}\right\}$ and $\left\{\tilde{r}_{t}^{b}\right\}$. These relationships do not generally apply to more elaborate versions of the Baseline formulation ( $\rho=0$ is necessary).

Let us in this context reinforce our earlier remarks concerning the commonplace characterizations of mean reversion. The results of Table 4.1 clearly suggest that to identify a mean reverting series exclusively with negative autocorrelation is not fully informative: all the series in Table 4.1 mean revert (they have finite $A C T$ measurements), yet only $\left\{\tilde{r}_{t}^{e}\right\}$ is negatively autocorrelated. 
Negative autocorrelation means "extremely frequent crossings of the mean" (a very small $A C T$ value) nothing more and the nature of these crossings can differ widely (Observation C).

We conclude this section with some summary remarks:

1. Multiple $A C T$ s map into the same autocorrelation measure, so that the latter is the coarser measure (and, therefore, less informative).

2. $A C T^{A}$ and $A C T^{B}$ provide more information as they directly measure the inverse of the frequency of transition.

3. Properties I and II, most especially, thus represent very restrictive characterizations of mean reversion.

The Baseline model falls short, however, of a full-fledged business cycle model on many dimensions. In particular, none of the aggregate series is sufficiently persistent vis-à-vis the data. In the next section we remedy this particular shortcoming and explore the consequences of this and other model generalizations for "mean reversion." 


\section{Model Generalizations}

In this section we explore how the $A C T^{A}, A C T^{B}, A C T$ and autocorrelation of security prices and returns are affected by adding a variety of model features to the Baseline formulation. It is well known that these added features, especially in combination, allow the Baseline model to approximate fairly well much of the observed statistical behavior of the U.S. economy's macro aggregate time series. In particular, we consider: (1) persistence in the productivity shocks, (2) incomplete depreciation, (3) greater concavity in the representative agent's period utility function, (4) habit formation, (5) the addition of a labor-leisure choice, and (6) factor share uncertainty. We describe in detail the consequences of (1) and (2) as these modifications have substantial implications. Because their effects are largely marginal, we only offer a brief summary of the results arising from (3) $-(6)$.

\subsection{Adding Persistence in the Productivity Shocks}

Table 5.1 and Proposition 5.2 illustrate the consequences of introducing persistence in the productivity shocks into the Baseline Model in a the way typical of the DSGE literature. We specialize the production technology to be of the form $y_{t}=\left(\tilde{k}_{t}\right)^{\alpha} e^{\tilde{\lambda}_{t}}$ where $\tilde{\lambda}_{t+1}=\rho \lambda_{t}+\tilde{\varepsilon}_{t+1} ;\left\{\tilde{\varepsilon}_{t}\right\}$ is i.i.d., $\tilde{\varepsilon}_{t} \sim N\left(0, \sigma_{\varepsilon}^{2}\right)$, and $\rho>0$. Even with persistence in the productivity shocks of this type, the decision rules take the same form as $(13)-(14) .^{23}$

\footnotetext{
${ }^{23}$ The necessary and sufficient conditions for the optimal investment function is: $u_{1}\left(c_{t}\right)=\beta \int u_{1}\left(\tilde{c}_{t+1}\right) \alpha k_{t+1}^{\alpha-1} e^{i_{t+1}} d F\left(\lambda_{t+1} ; \lambda_{t}\right)$. For the indicated functional forms and decision rules, this equation becomes:

$\frac{1}{(1-\alpha \beta) k_{t}^{\alpha} e^{\lambda_{t}}}=\beta \int \frac{\alpha\left(\alpha \beta k_{t}^{\alpha} e^{\lambda_{t}}\right)^{\alpha-1} e^{\rho \lambda_{t}+\tilde{\varepsilon}_{t+1}}}{(1-\alpha \beta)\left[\alpha \beta k_{t}^{\alpha} e^{\lambda_{t}}\right]^{\alpha} e^{\rho \lambda_{t}+\varepsilon_{t+1}}} d F\left(\tilde{\varepsilon}_{t+1}\right)$

which reduces to $1=\int d F\left(\tilde{\varepsilon}_{t+1}\right)=1$
} 
Furthermore, the addition of persistence also does not alter the expressions for $p_{t}^{e}$ and $r_{t}^{e}$. However, the expressions for $p_{t}^{b}$ and $r_{t}^{b}$ are modified as follows:

$$
p_{t}^{b}=\beta e^{\sigma_{\varepsilon}^{2} / 2} k_{t}^{\alpha-\alpha^{2}} e^{(1-\alpha-\rho) \tilde{\lambda}_{t}} /(\alpha \beta)^{\alpha} \text { with } r_{t}^{b}=1 / p_{t}^{b}-1 .
$$

As regards prices and dividends, the results mirror their earlier counterparts.

Proposition 5.1: For the Baseline model, where $\rho>0$ the equity price

series $\left\{\tilde{p}_{t}^{e}\right\}$, the dividend series $\left\{d_{t}\right\}$, and the risk free asset price series $\left\{\tilde{p}_{t}^{b}\right\}$ are all mean averting by Property I.

Proof: See the Technical Appendix.

Our analysis of returns relies on numerical simulations of (13) - (17). Panel A of Table 5.1 documents the correlations while panel B gives the corresponding $A C T \mathrm{~s}$; for representative cases the individual $A C T^{A}$ and $A C T^{B}$ are provided as well. 
Table 5.1

Model 2: Autocorrelations, $A C T_{\mathrm{s}}{ }^{(\mathrm{i})}$

$$
\begin{gathered}
u(c)=\log (c), \beta=.99, \Omega=1, \alpha=.36 \\
\tilde{\lambda}_{t+1}=\rho \lambda_{t}+\tilde{\varepsilon}_{t+1}, \sigma_{\varepsilon}=.00712^{(\mathrm{ii})}
\end{gathered}
$$

Panel A: Autocorrelations: Various $\rho$

$$
\rho=0 \quad \rho=.2 \quad \rho=.4 \quad \rho=.6 \quad \rho=.8 \quad \rho=.95
$$

\begin{tabular}{|c|c|c|c|c|c|c|}
\hline $\operatorname{ACT}\left(\tilde{p}_{t}^{e}\right)$ & 2.70 & 3.14 & 3.83 & 4.91 & 7.20 & 13.89 \\
\hline $\operatorname{ACT}^{A}\left(\tilde{p}_{t}^{e}\right)$ & 2.68 & & 3.80 & & & 13.18 \\
\hline $\operatorname{ACT}^{B}\left(\tilde{p}_{t}^{e}\right)$ & 2.71 & & 3.86 & & & 14.44 \\
\hline $\operatorname{ACT}\left(\tilde{p}_{t}^{b}\right)$ & 2.70 & 3.52 & 4.87 & 5.39 & 3.71 & 2.84 \\
\hline $\operatorname{ACT}^{A}\left(\tilde{p}_{t}^{b}\right)$ & 2.69 & & 4.88 & & & 2.82 \\
\hline $\operatorname{ACT}^{B}\left(\tilde{p}_{t}^{b}\right)$ & 2.71 & & 4.86 & & & 2.87 \\
\hline $\operatorname{ACT}\left(\tilde{r}_{t}^{e}\right)$ & 1.66 & 1.80 & 1.97 & 2.17 & 2.41 & 2.60 \\
\hline $\operatorname{ACT}^{A}\left(\tilde{r}_{t}^{e}\right)$ & 1.65 & & 1.96 & & & 2.60 \\
\hline $\operatorname{ACT}^{B}\left(\tilde{r}_{t}^{e}\right)$ & 1.69 & & 1.98 & & & 2.61 \\
\hline $\operatorname{ACT}\left(\tilde{r}_{t}^{b}\right)$ & 2.70 & 3.51 & 4.90 & 5.40 & 3.72 & 2.84 \\
\hline $\operatorname{ACT}^{A}\left(\tilde{r}_{t}^{b}\right)$ & 2.67 & & 4.84 & & & 2.85 \\
\hline $\operatorname{ACT}^{B}\left(\tilde{r}_{t}^{b}\right)$ & 2.71 & & 4.95 & & & 2.82 \\
\hline $\operatorname{ACT}\left(\tilde{r}_{t}^{p}\right)$ & 2.02 & 2.02 & 2.02 & 2.02 & 2.02 & 2.02 \\
\hline $\operatorname{ACT}^{A}\left(\tilde{r}_{t}^{p}\right)$ & 2.02 & & 2.02 & & & 2.02 \\
\hline $\operatorname{ACT}^{B}\left(\tilde{r}_{t}^{p}\right)$ & 2.02 & & 2.02 & & & 2.02 \\
\hline
\end{tabular}

$\begin{array}{lcccccc}\operatorname{corr}\left(\tilde{p}_{t}^{e}, \tilde{p}_{t+1}^{e}\right) & .39 & .54 & .68 & .80 & .90 & .98 \\ \operatorname{corr}\left(\tilde{p}_{t}^{b}, \tilde{p}_{t+1}^{b}\right) & .39 & .63 & .80 & .84 & .67 & .46 \\ \operatorname{corr}\left(\tilde{r}_{t}^{e}, \tilde{r}_{t+1}^{e}\right) & -.30 & -.16 & -.02 & .12 & .25 & .35 \\ \operatorname{corr}\left(\tilde{r}_{t}^{b}, \tilde{r}_{t+1}^{b}\right) & .39 & .63 & .80 & .84 & .68 & .46 \\ \operatorname{corr}\left(\tilde{r}_{t}^{p}, \tilde{r}_{t+1}^{p}\right) & .03 & .03 & .03 & .03 & .03 & .03\end{array}$

Panel B: $A C T \mathrm{~s}$

(i) Statistics based on the original base time series of length 10,000

(ii) The numbers reported in this table are unaffected by the magnitude of $\sigma_{\varepsilon}$. They are also unaffected by the choice of $\beta, 0<\beta<1$. 
While the results of Table 5.1-Panel A for equity returns mirror the conclusions of Propositions 3.3 for the $\rho=0$ case, they are not robust: sufficient persistence in the random productivity disturbance yields an equity return series that is mean averting (by Properties I and II). The other patterns are consistent with the conclusions of Proposition 4.2, negative autocorrelated series have $A C T \mathrm{~s}<2$, and Observation $\mathrm{F}$ : for all series $A C T \mathrm{~s}$ and correlations increase and decrease in tandem. This latter fact follows from the recognition that all the financial series evolve roughly symmetrically about their unconditional means, with the implication that their corresponding $T^{A B}$ matrices are close to symmetric.

The results of Table 5.1 for the $\left\{\tilde{r}_{t}^{e}\right\}$ series are partially rationalized in Proposition 5.2.

Proposition 5.2: Consider the model defined by (13) and (14) with production technology and shock process specialized to $y_{t}=k_{t}^{\alpha} e^{\tilde{\lambda}_{t}}$ where $\tilde{\lambda}_{t+1}=\rho \lambda_{t}+\tilde{\varepsilon}_{t+1},\left\{\tilde{\varepsilon}_{t}\right\}$ i.i.d. $N\left(0, \sigma_{\varepsilon}^{2}\right)$. A sufficient condition for bond and equity returns to be mean averting by Properties I and II is that $\alpha+\rho>1$.

Proof: See the Technical Appendix.

Proposition 5.2 argues that sufficiently persistent productivity disturbances in conjunction with production function concavity results in Property I $\left\{\tilde{r}_{t}^{e}\right\}$ mean aversion (risk free returns are always so). Therefore, if a model of this sort is to come close to matching the observed high persistence in output, equity returns will be mean averting by Property I and thus Property II. ${ }^{24}$

None of these results is surprising in the least: the process on the disturbance component, $\left\{e^{\bar{\lambda}_{t}}\right\}$, is itself highly mean averting (by Property I)

\footnotetext{
${ }^{24}$ This follows from Proposition 2.1.
} 
only if $\rho<0$, a selection inconsistent with the behavior of its counterpart, the Solow residual. For calibrations customary to the macro-finance literature $(\rho>0)$ mean aversion in equity returns results as well. It is not obvious what model features would allow high persistence in aggregate series (as the data reveals) to be compatible with mean reversion in equity returns and the equity premium, at least as characterized by Properties I and II. Cogley and Nason (1995) emphasize the close relationship of the properties of the productivity process to the derived properties of DSGE models' state variables and thus security prices and returns.

Proposition 5.3: Consider a stochastic process of the form $\tilde{x}_{t}=\rho x_{t-1}+\tilde{\varepsilon}_{t}$, where $\left\{\tilde{\varepsilon}_{t}\right\}$ is i.i.d. $N\left(0, \sigma_{\varepsilon}^{2}\right)$. Define a new stochastic process by

$$
\begin{aligned}
& \tilde{\lambda}_{t}=e^{\tilde{x}_{t}} \text {. Then, } \\
& \operatorname{cov}\left(\tilde{\lambda}_{t}, \tilde{\lambda}_{t+1}\right)\left\{\begin{array}{l}
>0 \text { if } 1>\rho>0 \\
<0 \text { if }-1<\rho<0
\end{array}\right\} .
\end{aligned}
$$

Proof: See the Technical Appendix ${ }^{25}$.

Proposition 5.3 bears upon two other unexpected features of Table 5.1. First, as productivity persistence $\rho$ increases, $\operatorname{corr}\left(\tilde{p}_{t}^{b}, \tilde{p}_{t+1}^{b}\right)$, while always positive, first increases (seeming to peak at $\rho=.6$ ) and then monotonically declines. The same pattern is observed for $\operatorname{corr}\left(\tilde{r}_{t}^{b}, \tilde{r}_{t+1}^{b}\right)$, although this is to be expected in view of the close association of $\left\{\tilde{p}_{t}^{b}\right\}$ and $\left\{\tilde{r}_{t}^{b}\right\}$ noted earlier. For the indicated shock process the price of the bond is specialized to the form indicated in footnote 26 and is composed of

\footnotetext{
${ }^{25}$ We thank Sergio Villar for his help in proving Proposition 5.3.
} 
three building clocks, the positive constant $\beta e^{\sigma_{\varepsilon}^{2} / 2} /(\alpha \beta)^{\alpha}$, a capital stock term $\tilde{k}_{t}^{\alpha-\alpha^{2}}$ and a productivity shock term $e^{(1-\alpha-\rho) \tilde{\lambda}_{t}}$. While the second term is mean averting for all values of $\rho$, the third term is Property I mean averting for $(1-\alpha-\rho)>0$, and Property I mean reverting for $(1-\alpha-\rho)<0$ by Proposition 5.3 with the switch occurring for $\rho$ slightly greater than .6 (since $\alpha=.36$ ). Thus for low values of $\rho$, the mean aversion across the two terms is reinforcing as $\rho$ increases; for $\rho$ exceeding .64, the (Property I) mean reversion of the third term works against the (Property I) mean aversion of the second to bring about the observed effect. We lack an intuitive explanation.

The second curious fact is the near independence of the $\left\{\tilde{r}_{t}^{p}\right\}$ series, as identified by $A C T_{\left\{\tilde{r}_{t}^{p}\right\}}^{A}=A C T_{\left\{\tilde{r}_{t}^{p}\right\}}^{B}=2.02$ (since this measurement includes the period of crossing, the average number of periods strictly above and strictly below the mean are individually each about one). In fact, this near independence is robust to all the model modifications considered in this paper.

Turning to Property III, as shock persistence increases $(\rho>0)$ our earlier Property III results (Proposition 3.4) are weakened for prices: Table 5.2 summarizes the results of extensive numerical simulations that compute $\operatorname{corr}\left(\tilde{x}_{s}-\tilde{x}_{r}, \tilde{x}_{u}-\tilde{x}_{r}\right)$ for a wide class of $\{r, s, t, u\}$, where $r<s<t<u$, and $\left\{\tilde{x}_{t}\right\}$ is chosen from $\left\{\tilde{r}_{t}^{e}\right\},\left\{\tilde{r}_{t}^{p}\right\}$, and $\left\{\tilde{p}_{t}^{e}\right\}$ 


\section{Table 5.2}

\section{Correlations: Various Series}

$$
\begin{aligned}
& u(c)=\log (c), \beta=.99, \Omega=1, \alpha=.36 \\
& \tilde{\lambda}_{t+1}=\rho \lambda_{t}+\tilde{\varepsilon}_{t+1}, \sigma_{\varepsilon}=.00712, \rho=.95
\end{aligned}
$$

Simulation Results for $\mathrm{s}=\mathrm{r}+\mathrm{i}, \mathrm{t}=\mathrm{s}+\mathrm{j}, \mathrm{u}=\mathrm{t}+\mathrm{k}$

$$
\mathrm{i}, \mathrm{j}, \mathrm{k} \in\{1,2,3\}
$$

Series Correlation

(i) $\operatorname{corr}\left(\tilde{p}_{s}^{e}-\tilde{p}_{r}^{e}, \tilde{p}_{u}^{e}-\tilde{p}_{t}^{e}\right)$ ambiguous

(ii) $\operatorname{corr}\left(\tilde{r}_{s}^{e}-\tilde{r}_{r}^{e}, \tilde{r}_{u}^{e}-\tilde{r}_{t}^{e}\right)<0$

(iii) $\operatorname{corr}\left(\tilde{r}_{s}^{p}-\tilde{r}_{r}^{p}, \tilde{r}_{u}^{p}-\tilde{r}_{t}^{p}\right)<0$
Range of Values across all i, j, k

These results are largely inconsistent with earlier results concerning $\left\{\tilde{r}_{t}^{e}\right\},\left\{\tilde{r}_{t}^{p}\right\}$, and $\left\{\tilde{p}_{t}^{e}\right\}$ for Property I (see Table 5.1, right most column), a fact that accounts for our earlier comment that Property III represents a fundamentally different measurement from either Property I or II. ${ }^{26}$

We close Section 5.1 with a summary of what we have learned: First, persistence in the productivity disturbance generically overturns specific results relative to the case of independence: equity returns appear necessarily to be Property I mean reverting only in the presence of low persistence productivity disturbances. Proposition 5.3 further suggests that this particular phenomenon is likely to be pervasive across many DSGE formulations, implying that the search for mean reversion in equity returns and the equity premium, at least as characterized by Properties I and II, and III, is unlikely to be fruitful - if the present family of models

\footnotetext{
${ }^{26}$ For this reason it deserves greater recognition and evaluation. The patterns in Table 5.2 are largely consistent with Proposition 3.4. We thank Natalia Gershun for her many contribution to Table 5.2.
} 
has anything to say about actual economies. To put it differently, we find it unsurprising from a theoretical perspective, that evidence for mean reversion in historical equity returns is weak if, indeed, it is based on the economic fundamentals emphasized in the present, simplified macro-analysis.

\subsection{Incomplete Depreciation}

In this section we modify the model of Section 5.1 to admit partial depreciation. As a result, the equation of motion on capital stock becomes:

$$
k_{t+1}=(1-\Omega) k_{t}+i_{t} .
$$

With this change, closed form expressions for the risk-free bond price and its rate of return are not available. We therefore continue to rely exclusively on numerical simulation. Table 5.3 summarizes the results. 
Table 5.3

Autocorrelations, $A C T \mathrm{~s}$

Baseline Case: $u(c)=\log (c), \alpha=.36, \beta=.96, \sigma_{\varepsilon}^{2}=.00712$

Various $\boldsymbol{\Omega}, \boldsymbol{\rho}$

\begin{tabular}{lcccccccccc} 
& \multicolumn{9}{c}{ Panel A: Autocorrelations } \\
& \multicolumn{9}{c}{$\Omega=1$} & \multicolumn{3}{c}{$\Omega=.4$} & & $\Omega=.025$ \\
& $\rho=0$ & $\rho=.4$ & $\rho=.95$ & $\rho=0$ & $\rho=.4$ & $\rho=.95$ & $\rho=0$ & $\rho=.4$ & $\rho=.95$ \\
$\operatorname{corr}\left(\tilde{p}_{t}^{e}, \tilde{p}_{t+1}^{e}\right)$ & 0.39 & 0.68 & 0.98 & 0.67 & 0.84 & 0.99 & 0.97 & 0.98 & 1.00 \\
$\operatorname{corr}\left(\tilde{p}_{t}^{b}, \tilde{p}_{t+1}^{b}\right)$ & 0.39 & 0.80 & 0.46 & 0.67 & 0.87 & 0.67 & 0.97 & 0.60 & 0.93 \\
$\operatorname{corr}\left(\tilde{r}_{t}^{e}, \tilde{r}_{t+1}^{e}\right)$ & -0.30 & -0.02 & 0.35 & -0.13 & 0.20 & 0.63 & 0.02 & 0.41 & 0.93 \\
$\operatorname{corr}\left(\tilde{r}_{t}^{b}, \tilde{r}_{t+1}^{b}\right)$ & 0.39 & 0.80 & 0.46 & 0.67 & 0.87 & 0.67 & 0.97 & 0.60 & 0.93 \\
$\operatorname{corr}\left(\tilde{r}_{t}^{p}, \tilde{r}_{t+1}^{p}\right)$ & 0.03 & 0.03 & 0.03 & 0.03 & 0.03 & 0.03 & 0.03 & 0.03 & 0.03 \\
$\operatorname{corr}\left(\tilde{c}_{t}, \tilde{c}_{t+1}\right)$ & 0.39 & 0.68 & 0.98 & 0.67 & 0.82 & 0.98 & 0.97 & 0.98 & 1.00 \\
$\operatorname{corr}\left(\tilde{k}_{t}, \tilde{k}_{t+1}\right)$ & 0.39 & 0.68 & 0.98 & 0.67 & 0.84 & 0.99 & 0.97 & 0.98 & 1.00
\end{tabular}

Panel B: $A C T$ s

$\begin{array}{lccccccccc}\operatorname{ACT}\left(\tilde{p}_{t}^{e}\right) & 2.696 & 3.827 & 13.89 & 3.77 & 5.49 & 19.92 & 11.31 & 16.89 & 74.63 \\ \operatorname{ACT}\left(\tilde{p}_{t}^{b}\right) & 2.697 & 4.876 & 2.84 & 3.78 & 6.11 & 3.81 & 11.19 & 3.35 & 8.51 \\ \operatorname{ACT}\left(\tilde{r}_{t}^{e}\right) & 1.658 & 1.968 & 2.61 & 1.83 & 2.32 & 3.58 & 2.01 & 2.737 & 8.33 \\ \operatorname{ACT}\left(\tilde{r}_{t}^{b}\right) & 2.691 & 4.898 & 2.84 & 3.77 & 6.07 & 3.81 & 11.22 & 3.35 & 8.52 \\ \operatorname{ACT}\left(\tilde{r}_{t}^{p}\right) & 2.02 & 2.02 & 2.02 & 2.02 & 2.02 & 2.02 & 2.02 & 2.02 & 2.02\end{array}$


The message of Panels A and B of Table 5.3 is unambiguous: lower depreciation rates (smaller $\Omega$ ) increase autocorrelations for all price and returns series except the premium, which is largely unaffected. Compatible results are found in the $A C T$ measurements. When $\Omega=.025$ all series become positively autocorrelated, even for $\left\{\tilde{r}_{t}^{e}\right\}$ when $\rho=0$, in contrast to the conclusions of Proposition 3.3 (which applies only to the $\Omega=1$ case).

Why is this observed? When the depreciation rate declines, then, ceteris paribus, the period $t+1$ capital stock becomes more similar to its period t predecessor. At the same time, investment, which is volatile, shrinks as a proportion of period $t+1$ capital. The consumption series is similarly affected. As a result, both capital stock and consumption become more highly autocorrelated as indicated at the bottom of Panel A. If capital stock becomes more highly autocorrelated so also must be the series $\left\{\tilde{p}_{t}^{e}\right\}$ and $\left\{\tilde{r}_{t}^{e}\right\}$. If consumption becomes more highly autocorrelated, so will the risk-free bond price series and the risk-free return. The lack of consequences for the premium. 


\subsection{Other Parameter Changes}

As mentioned earlier, we also explored the consequences of increasing risk aversion ( directly or indirectly, via external habit formation), the addition of endogenous labor/leisure choice etc. These results are summarized below; the underlying tables and the full explanatory text can be found in the on-line Appendix. In all cases the conclusions reported are based on the values (entry by entry) associated with the same parameters $\left(\alpha, \beta, \rho, \Omega, \sigma_{t}^{2}\right)$ as employed in Table 5.2.

\subsubsection{Greater Risk Aversion ${ }^{27}$}

Entry by entry we see that higher risk aversion, ceteris paribus, increases autocorrelations and $A C T$ s across all return and price series except for the premium which is unaffected. Greater representative agent risk aversion translates into the desire for a smoother intertemporal consumption stream, which leads to higher consumption autocorrelation. It directly follows that the price of one unit of consumption next period, the risk-free bond price, and its associated return will become more highly autocorrelated as well.

On the equity side, in order to promote a smoother consumption path, the path of the capital stock, $\left\{p_{t}^{e}\right\}$ must be made intertemporally more stable - more positively autocorrelated at the expense of greater investment volatility (to which the representative agent is indifferent). Accordingly, the equity price and return series becomes more highly autocorrelated. In summary, within the CRRA class of preference orderings, greater risk aversion promotes Property I mean aversion for all financial series; ACT patterns follow in tandem.

It is well known that habit formation causes the agent to behave in a more risk averse fashion ${ }^{28}$. Following the conclusions earlier in this section, we find that the

\footnotetext{
${ }^{27}$ Here we expand the basic model to include period preference orderings captured by $u\left(c_{t}\right)=c_{t}^{1-\gamma} / 1-\gamma$, for various $\gamma>1$.
} 
addition of habit formation increases autocorrelations and ACTs across the board (all cases of returns and prices except for the premium). The logic behind this effect is also unchanged from earlier. Higher degrees of risk aversion further compound the effect.

\subsubsection{Adding a Labor/Leisure Choice ${ }^{29}$}

First, if $\Omega=1$, then the addition of a labor/leisure choice under either specification has no impact on the autocorrelations or $A C T$ s for any of the financial series we study. If $\Omega<1$, then the addition of a labor/leisure choice slightly diminishes the autocorrelations and $A C T$ s for all the series.

In the cases where $\Omega=1$, the equilibrium level of hours worked, $n_{t}$ is independent of the shock and capital stock values. The extent of hours represents a level effect alone, and the $A C T$ s are thus unaffected. In the cases where $\Omega<1$, the fact that the $A C T$ s are all somewhat diminished indicates that the addition of a labor decision variable tends to pull the capital stock and consumption series back towards their means, relative to an environment in which it is absent. The effect is very modest, however, and greatest in the $\Omega=.025$ cases where the decline in $A C T$

${ }^{28}$ In the Baseline case, this means modifying the representative agent's period utility function to be of the form $u\left(c_{t}-\psi c_{t-1}\right)=\log \left(c_{t}-\psi c_{t-1}\right)$; with higher risk aversion, CRRA utilities are modified similarly

${ }^{29}$ We do this in two related ways by specifying the representative agent's period utility function to be either

$$
u\left(c_{t}, 1-n_{t}\right)=\log c_{t}+A \log \left(1-n_{t}\right)
$$

or

$$
\text { (2) } \quad u\left(c_{t}, 1-n_{t}\right)=\left(c_{t}^{\delta}\left(1-n_{t}\right)^{1-\delta}\right)^{1-\gamma} / 1-\gamma
$$

where, $n_{t}$ is the hours of labor supplied in period $t$. In either case the production function is generalized to be of the form

$f\left(k_{t}, n_{t}\right) e^{\tilde{\lambda}_{t}}=k_{t}^{\alpha}\left(n_{t}\right)^{1-\alpha} e^{\tilde{\lambda}_{t}}$ 
magnitude is about $10 \%$. This is a way of saying that variations in the supply of the agent's labor do assist in stabilizing both the economy's capital stock series (and thus reduce the $A C T$ s of $\left\{\tilde{p}_{t}^{e}\right\}$ and $\left\{r_{t}^{e}\right\}$ ), and its consumption series (and thus reduce the $A C T$ s of $\left\{\tilde{p}_{t}^{b}\right\}$ and $\left.\left\{r_{t}^{b}\right\}\right)$, a fact well known in the business cycle literature. The effect is small, however, not only because the agent also prefers low variation in leisure, $\left(1-n_{t}\right)$, but also for the fact that the capital stock and hours series are themselves very highly positively correlated. The $A C T$ s exceed 2.

We summarize these observations as follows:

1. For the representative agent class of models and the standard mean reversion characterization, mean aversion, as defined by Properties I and II, in equity and risk free return is the norm, except in the Baseline case where $\Omega=1$, and shock correlation is low.

2. Conditional on the same levels of $\Omega$ and $\rho$, the previous sections demonstrate that the addition (individually or collectively) of a wide variety of model features to the Baseline paradigm only serves to increase the Property I--II mean aversion in most of the time series of interest (the premium is the exception). If the source of uncertainty is a multiplicative productivity shock, we venture to suggest that this feature will generally be observed, whatever additional features are imposed.

3. The equity premium remains slightly Property I mean averting for the wide class of models that we have studied.

In short, Property I or II "mean aversion" appears to rule for all financial time series within this family of DSGE models. This must be the case if the models are to have empirical relevance. 


\subsection{Factor Share Uncertainty}

The decision to introduce factor share uncertainty arises from the results reported in Guvenen (2009). He observes a mild negative autocorrelation in the equity premium at lags of $1,2,3,5$ and 7 years arising in an incomplete markets model where shareholders trade both equity and debt, but workers trade only debt securities, a restriction that generates time varying income shares to these two groups. Following Lansing (2015), we capture variation in factor shares by a reduced form model where uncertainty arises via a stochastic production parameter $\tilde{\alpha}$. In the family of complete market models we have been considering, $\left\{\alpha_{\mathrm{t}}\right\}$ represents the share of income to capital ${ }^{30}$.

For all indicated parameter combinations, both the correlations and the corresponding $A C T$ 's are, by and large, similar in the two tables except for the mild

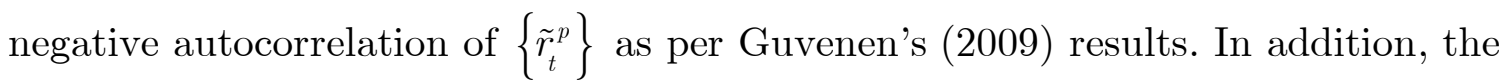
return on equity is very slightly mean averting for all $\rho$ values unlike in the Baseline case, and the monotonicity in equity return autocorrelations and $A C T$ s observed in the Baseline case is lost. Otherwise, if uncertainty in the capital share parameter is

\footnotetext{
${ }^{30}$ In particular, we explore the extent of mean reversion in the simple complete markets model identified by the following optimum formulation:
}

$$
\begin{aligned}
& \max _{\left\{z_{t}\right\}} E\left(\sum_{t=0}^{\infty} \beta^{t} u\left(c_{t}\right)\right) \\
& c_{t}+z_{t} \leq k_{t}^{\tilde{\alpha}_{t}} \\
& k_{t+1}=(1-\Omega) k_{t}+z_{t} \\
& \tilde{\alpha}_{t}=\rho \alpha_{t-1}+\tilde{\varepsilon}_{t}, \tilde{\varepsilon}_{t} \sim N\left(0, \sigma_{\varepsilon}^{2}\right)
\end{aligned}
$$

Note that the optimal decision rules for the model of this footnote are identical to those for the Baseline formulation. 
introduced into model (6) does not radically alter the correlation and ACT structure revealed in the Baseline formulation.

We next explore average crossing times for several important US financial time series.

\section{Empirical Support}

Table 6 presents the ACTs and autocorrelations for a representative collection of U.S. financial return time series. They are: AGG (Barclays Aggregate Bond Fund), EEM (MSCI Emerging Markets Index ETF), SPY (SPDR S\&P 500 ETF), GLD (SPDR Gold Shares), USO (United States Oil Fund ETF), TIP (Barclays TIPS Bond Fund ETF), VTI (Vanguard Total Stock Market EFT), VNQ (Vanguard Real Estate EFT) and VIX (CBOE Volatility Index). We provide the data at daily, monthly, and quarterly frequencies. The quarterly series best corresponds to the implicit time

period in the model economies. Nevertheless, all three frequencies should generate $A C T$ and autocorrelation relationships generally in accordance with Proposition 4.2 and Observations A. - F. 
Table 6.1

Average Crossing Times, Autocorrelations and Conditional Probabilities: Various Financial Return Series

\begin{tabular}{|c|c|c|c|c|c|c|c|}
\hline \multirow[b]{2}{*}{ Series $^{(i)}$} & \multicolumn{6}{|c|}{ Panel A: Quarterly Frequency } & \multirow[b]{2}{*}{$\phi^{B B}$} \\
\hline & Data Period & $A C T$ & $A C T^{A}$ & $A C D^{B}$ & $\rho$ & $\phi^{A A}$ & \\
\hline SPY & $2.1993-4.2017^{(\mathrm{ii})}$ & 2.3 & 2.61 & 2 & .092 & .62 & .5 \\
\hline EEM & $3.2003-4.2017$ & 2.23 & 2.31 & 2.15 & .207 & .57 & .54 \\
\hline $\mathrm{AGG}$ & $4.2003-4.2017$ & 1.9 & 1.87 & 1.93 & -.21 & .46 & .48 \\
\hline TIP & $1.2004-4.2017$ & 1.87 & 1.87 & 1.87 & $.05^{\text {(iii) }}$ & .46 & .46 \\
\hline VNQ & $4.2004-4.2017$ & 2.30 & 2.25 & 2.36 & .15 & .56 & .57 \\
\hline GLD & $1.2005-4.2017$ & 1.86 & 1.86 & 1.86 & $.04^{(\mathrm{iii})}$ & .46 & .46 \\
\hline VTI & $3.2001-4.2017$ & 2.13 & 2.53 & 1.75 & .12 & .61 & .43 \\
\hline USO & $3.2006-4.2017$ & 2 & 2.27 & 1.75 & .14 & .56 & .43 \\
\hline VIX & $2.1990-4.2017$ & 1.82 & 1.43 & 2.19 & -.31 & .30 & .54 \\
\hline
\end{tabular}

(i) The series corresponding to these abbreviations are found in the text

(ii) The notation 2.1993 indicates the second quarter of 1993, etc.

(iii) Indicates departures from theory.

\begin{tabular}{|c|c|c|c|c|c|c|c|}
\hline \multicolumn{8}{|c|}{ Panel B: Monthly Frequency } \\
\hline Series & Data Period & $A C T$ & $A C T^{A}$ & $A C D^{B}$ & $\rho$ & $\phi^{A A}$ & $\phi^{B B}$ \\
\hline SPY & $02.1993-12.2017^{(\mathrm{i})}$ & 2.02 & 2.26 & 1.78 & .068 & .56 & .44 \\
\hline EEM & $05.2003-12.2017$ & 2 & 2.05 & 1.95 & .12 & .51 & .49 \\
\hline AGG & $10.2003-12.2017$ & 2.16 & 2.21 & 2.13 & .04 & .55 & .53 \\
\hline TIP & $01.2004-12.2017$ & 1.78 & 1.77 & 1.81 & -.03 & .43 & .45 \\
\hline VNQ & $10.2004-12.2017$ & 1.96 & 2.10 & 1.82 & $.05^{(\mathrm{ii})}$ & .52 & .45 \\
\hline GLD & $12.2004-12.2017$ & 1.99 & 1.97 & 2 & -.11 & .49 & .5 \\
\hline VTI & $06.2001-12.2017$ & 2.09 & 2.40 & 1.79 & .16 & .58 & .44 \\
\hline USO & $05.2006-12.2017$ & 2.09 & 2.18 & 2 & .29 & .54 & .5 \\
\hline VIX & $02.1990-12.2017$ & 1.73 & 1.47 & 2 & -.16 & .32 & .0 \\
\hline
\end{tabular}

(i) The notation 02.1993 , signifies the $2^{\text {nd }}$ month of 2003 , etc.

(ii) Indicates departure from theory.

Panel C: Daily Frequency

$\begin{array}{lcccccr}\text { Series } & \text { Data Period } & A C T & A C T^{A} & A C D^{B} & \rho & \phi^{A A} \\ \text { SPY } & 02.01 .1993-12.29 .2017^{(\mathrm{i})} & 1.92 & 1.97 & 1.87 & -.06 & .49 \\ \text { EEM } & 04.14 .2003 \backslash-12.29 .2017 & 1.95 & 2.02 & 1.88 & -.10 & .51 \\ \text { AGG } & 09.29 .2003-12.29 .2017 & 1.85 & 1.89 & 1.81 & -.12 & .47 \\ \text { TIP } & 12.08 .2003-12.29 .2017 & 1.96 & 1.98 & 1.96 & .01^{(i i)} & .50 \\ \text { VNQ } & 09.30 .2004-12.29 .2017 & 1.96 & 2.00 & 1.91 & -.18 & .50 \\ \text { GLD } & 11.19 .2004-12.29 .2017 & 1.92 & 1.96 & 1.87 & -.02 & .49 \\ \text { VTI } & 06.01 .2001-12.29 .2017 & 1.95 & 2.04 & 1.85 & -.06 & .51 \\ \text { USO } & 04.11 .2006-12.29 .2017 & 1.93 & 1.96 & 1.89 & -.05 & .49 \\ \text { VIX } & 01.03 .1990-12.29 .2017 & 1.99 & 1.81 & 2.15 & -.08 & .47 \\ \end{array}$

(i) 02.01 .1993 is to be read as February 1, 1993, etc.

(ii) Indicates departure from theory. 
There are a number of relevant observations. First, note that at quarterly frequencies (Panel A), the majority (5 out of 9 ) of return series are Property I mean averting as was the case for all the models of Section 5, when they were subject to empirically relevant productivity autocorrelations. Unlike the present models, empirical bond returns (the AGG series) are mean reverting; this is also true of the VIX although it has no counterpart in the model-generated return series. In contrast, all series but one are Property I mean reverting at daily frequency; the sole exception (the TIPS series) being essentially independently distributed on a set basis through time (note that $\phi^{A A}=\phi^{B B}$ ). Nothing here is surprising; it is to be expected that the underlying processes governing daily returns, whatever they are, will be largely unrelated to an economy's aggregate investment and consumption processes.

By Proposition 4.2, if $A C T \leq 2$, Property I mean reversion should be observed. At daily frequencies, this is the case for all series except for TIPs. For monthly and quarterly series the exceptions are TIPs and GLD (quarterly) and VNG (monthly). Due to the relative lack of data at quarterly frequencies, it is not entirely surprising that the greatest number of inconsistencies are found there. At all frequencies the VIX series strongly endorses the theory.

Note that at daily frequencies all the corresponding $T$ matrices are not far off independence with the VIX the possible exception. At monthly and quarterly frequencies, however, this is generally not so, with the VIX return series again being the most asymmetric in both cases; the VTI is the next most extreme in this regard. In particular, at quarterly frequencies, the VIX has a .30 probability $\left(\phi^{A A}=.3\right)$ of remaining in the above-mean state, while only experiencing a mildly less-than-even chance of returning to it $\left(1-\phi^{B B}=.46\right)$. At quarterly frequencies the VTI series remains in the above mean state with probability .61 and returns to it only with probability .57. As a result, it has a high $A C T^{A}$. By comparison the $A C T$ for the VIX 
is the lowest of all the series for all data frequencies. We leave the rationale for this pattern to those more familiar with its underlying determination.

Observation E makes clear that there is no necessary positive association between an increasing $A C T$ and an increasing autocorrelation across the series, and we do not observe it in the data. With $A C T$ s around 2 (all of our series) the range of (ACT, correlation) possibilities is large (see Figures 1 and 2). Nevertheless, there is a weak positive association between a series' $A C T$ and its autocorrelation, an assertion captured in Figure 3, where we undertake the following simple cross-sectional regression:

$$
\text { autocorrelation }^{i}=\alpha+\beta A C T^{i}+\tilde{\varepsilon}_{i} .
$$

We find $\beta>0$ with an $\mathrm{R}^{2}$ of .0656 for monthly series and a $\mathrm{T}$ statistic of .65, which is unfortunately not significant. We suspect this is due to the high dispersion in the estimates; the results are similar at the other frequencies we consider. The fact that the $\mathrm{R}^{2}$ is low derives from the observation that many of the series have set transition matrices that are far from symmetric, as manifest in $\emptyset^{A A}$ being significantly different from $\emptyset^{B B}$ (and, as a result, $A C T^{A}$ and $A C T^{B}$ differing considerably). This being said, the modest theory presented in Section 4 finds modest empirical support. 


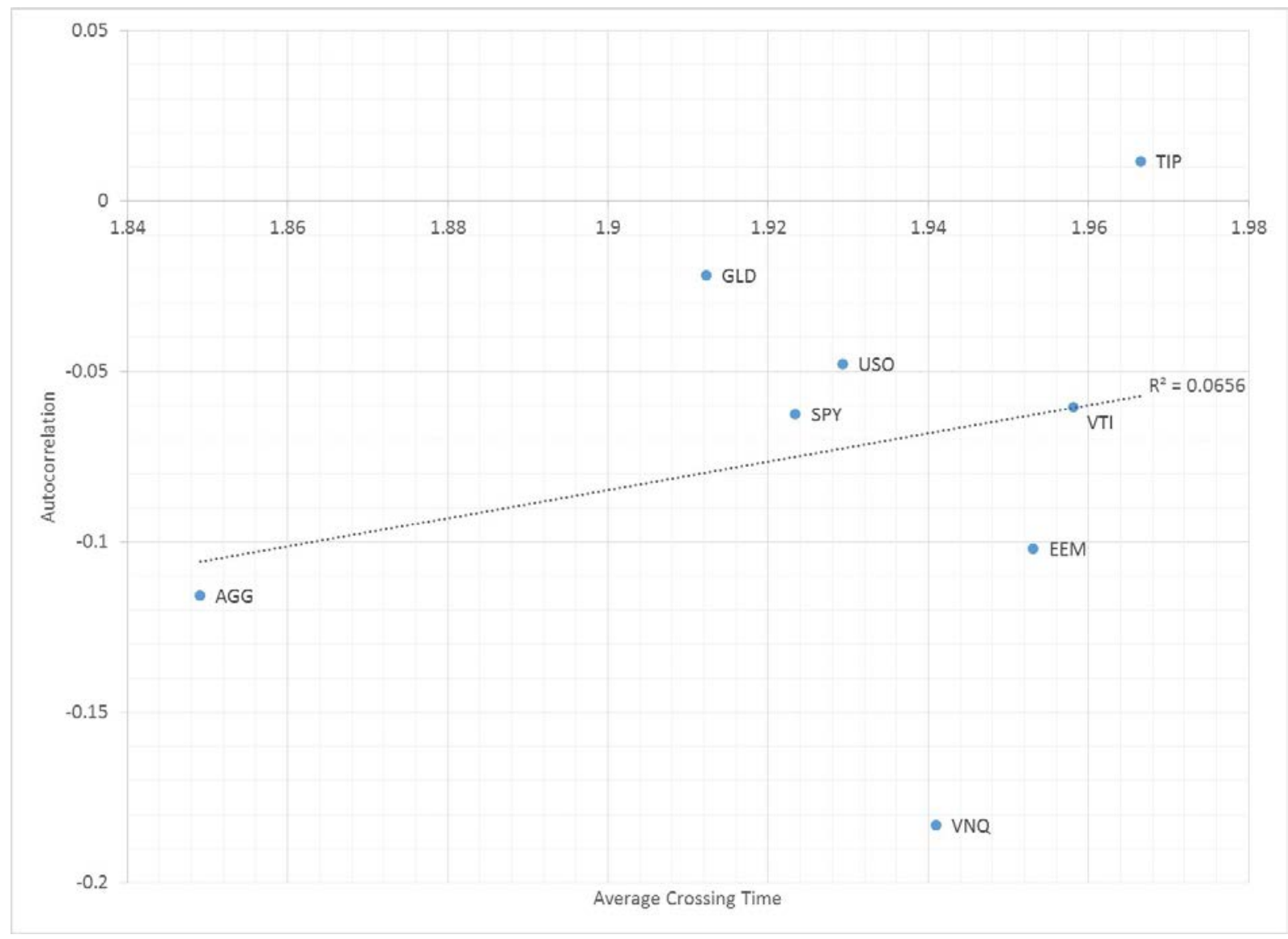

Figure 3

Autocorrelation vs. $A C T$

\section{Conclusion}

In this paper, we have argued that the notions of mean reversion and mean aversion can be synthesized under one metric, the Average Crossing Time $(A C T)$ with $A C T^{A}$ and $A C T^{B}$ as its underlying constituents. By the $A C T$ measure, the mean aversion/reversion distinction is entirely artificial, with a mean reverting (stationary) process being only identified with a finite $A C T$ value. One may think of mean averting processes as those with larger $A C T$ s but there is nothing in the $A C T$ concept that specifies a mean reversion/aversion demarcation value. The $A C T$ concept does provide, however, a simple, intuitive sense of one time series being more strongly 
mean reverting than another: its $A C T^{A}$ and $A C T^{B}$ are each lower than its comparison counterparts.

As an identifying measure, the $A C T$ allows us to evaluate other time series characteristics that have been "traditional identifiers" of "mean reversion." We considered four of these, classifying them as Properties I - IV. Properties I and II were shown to be satisfied in the case of the $A C T$ being less than or equal to two, which strikes us as an extremely strong criterion for "mean reversion." Most of the analysis in the paper concerns these properties, as they are the most widely employed. A careful analysis of Property III is left to future work, for two reasons. First it does not discriminate in any way for the canonical "mean reverting" $\mathrm{AR}(1)$ process across autocorrelation parameters. Second, it is not often seen. The same should be said for Property IV; it is very restrictive, not being satisfied in the traditional stationary models such as those reviewed in Section 5 .

Is there any real mean reversion/aversion distinction regarding stationary time series? Our analysis suggests the distinction is purely arbitrary. 


\section{References}

Basu, P. and P. Samanta, "Volatility and Stock Prices: Implications from a Production Model of Asset Pricing," Economics Letters, 70 (2001), 229-235.

Basu, P. and H. Vinod, "Mean Reversion in Stock Prices: Implications from a Production Based Asset Pricing Model," The Scandanavian Journal of Economics, 96 (1994), 51-65.

Bekaert, G., and R.J. Hodrick, International Financial Management, $3^{\text {rd }}$ Edition (2017), Cambridge University Press.

Bessembinder, H., J. Coughenour, P. Seguin, and M. Monroe, "Mean Reversion in Equilibrium Asset Prices: Evidence from the Futures Term Structure," Journal of Finance, 50 (1995), 361-375.

Bossaerts, P., and P. Hillion, "Implementing Statistical Criteria to Select Return Forecasting Models: What Do We Learn?", Review of Financial Studies, 12 (1999), 405-428.

Brock, W.A., "An Integration of Stochastic Growth Theory and the Theory of Finance," in McCall, J.J. (Ed.), The Economics of Information and Uncertainty (1982), Chicago: University of Chicago Press.

Campbell, J., and N. G. Mankiw, "Are Output Fluctuations Transitory?", Quarterly Journal of Economics, 102 (1987), 857-880.

Campbell, J., and R. Shiller, "The Dividend Price Ratio and the Expectations of Future Dividends and Discount Factors," Review of Financial Studies, 1 (1988), 195-227.

Campbell, J., and M. Yogo, "Efficient Tests of Stock Return Predictability," Journal of Financial Economics, 81 (2006), 27-60. 
Cecchetti, S., P. Lam, and N. Mark, "Mean Reversion in Equilibrium Asset Prices," American Economic Review, 80 (1990), 398-418.

Cochrane, J., "Presidential Address," The Journal of Finance, 66 (2011), 1047-1108.

Cogley, T., and J. Nason, "Output Dynamics in Real Business Cycle Models," The American Economic Review, 85 (1995), 492-511.

Daniel, K., "The Power and Size of Mean Reversion Tests," Journal of Empirical Finance, 8 (2001), 493-535.

Donaldson, J. B., N. Gershun, and R. Mehra, "Notes on Mean Reversion from a DSGE Perspective," Working Paper, Columbia University (2015).

Exley, J., S. Mehta, and A. Smith, "Mean Reversion," Faculty \& Institute of Actuaries Finance and Investment Conference Brussels, June 2004 Downloaded from https://www.actuaries.org.uk/documents/mean-reversion

Fama, E. F. and K. R. French, "Permanent and Temporary Components of Stock Prices," Journal of Political Economy, 96 (1988), 246-273.

Fortunin, C., P. Kasteleyn, and J. Ginibre, "Correlation Inequalities on Some Partially Ordered Sets," Communications in Mathematical Physics, 22 (1971), 89-103.

Goyal, A., and I. Welch, "Predicting the Equity Premium with Dividend Ratios," Management Science, 49 (2003), 639-654.

Guvenen, F., "A Parsimonious Model for Asset Pricing," Econometrica, 77 (2009), $1711-1750$.

Harris, T., "A Lower Bound for the Critical Probability in a Certain Percolation," Proceedings of the Cambridge Philosophical Society, 56 (1960), 13-20.

Hopenhayn, H., and E.C. Prescott, "Stochastic Monotonicity and Stationary Distributions for Dynamic Economies," Econometrica, 60 (1992), 1387-1406. Kamihigashi, T., and J. Stachurski, "Stochastic Stability in Monotone Economies," Journal of Theoretical Economics, 9 (2014), 383-407. 
Kim, C.J. and C.R. Nelson, "Testing for Mean Reversion in Heteroskedastic Data II: Autoregression Tests Based on Gibbs-Sampling-Augmented Randomization," Journal of Empirical Finance, 5 (1998), 385-396.

Kim, M., C.R. Nelson, and R. Startz, "Mean Reversion in Stock Prices? A Reappraisal of the Empirical Evidence," The Review of Economic Studies, 58 (1991), 515-528.

Lansing, K.J., "Asset Pricing with Concentrated Ownership and Distribution Shocks," American Economic Journal: Macroeconomics, 7 (2015), 67-103

Lewellen, J., "Predicting Returns with Financial Ratios," Journal of Financial Economics, 74 (2004), 209-235.

Lo, A. W., and A. G. MacKinlay, "Stock Market Prices do not Follow Random Walks: Evidence from a Simple Specification Test," Review of Financial Studies, 1 (1988), 41-66.

Lucas, R.E., Jr., "Asset Prices in an Exchange Economy," Econometrica, 66 (1978), 1429-1445.

Mukherji, S., "Are Stock Returns Still Mean Reverting?", Review of Financial Economics, 20 (2011), 22-27.

Poterba, J.M. and L.H. Summers, "Mean Reversion in Stock Prices: Evidence and Implications," Journal of Financial Economics, 22 (1988), 27-59.

Prescott, E.C., and R. Mehra, "Recursive Competitive Equilibrium: The Case of Homogeneous Households," Econometrica, 48 (1980), 1365-1379.

Richardson, M., and J.H. Stock, "Drawing Inferences from Statistics Based on MultiYear Asset Returns," Journal of Financial Economics, 25 (1989), 323-348.

Spierdijk, L., and J. Bikker, "Mean-Reversion in Stock Prices: Implications for Long Term Investors," De Nederlandsche Bank NV Working Paper \#343, April 2012. 
Summers, L.H., "Does the Stock Market Rationality Reflect Fundamental Values?", Journal of Finance, 41 (1986), 591-601.

Torous, W., R. Valkanov, and S. Yau, "On Predicting Stock Returns with Nearly Integrated Explanatory Variables," Journal of Business, 77 (2004), 937-966.

Welch, I., and A. Goyal, "A Comprehensive Look at the Empirical Performance of Equity Premium Predictions," Review of Financial Studies, 21 (2008), 14551508.

Zakamulin, V., "Secular Mean Reversion and Long-Run Predictability of the Stock Market," Working Paper, University of Agder, Norway (2015). 


\section{Technical Appendix}

In this appendix we extensively use a special case of the Fortunin, Kasteleyn and Ginibre (1971) inequality ${ }^{1}$ (henceforth FKG) in Harris (1960). This inequality (adapted to our setting) states that for any probability measure on $R$, and increasing

functions $f(x)$ and $g(x), \int_{R} f(x) g(x) d \mu(x) \geq \int_{R} f(x) d \mu(x) \int_{R} g(x) d \mu(x)$.

\section{Proof of Proposition 2.1}

a. $\quad$ Property II $\Rightarrow$ Property I

$$
\begin{gathered}
\operatorname{var}\left(\tilde{x}_{t}+\tilde{x}_{t+1}\right)=\operatorname{var}\left(\tilde{x}_{t}\right)+\operatorname{var}\left(\tilde{x}_{t+1}\right)+2 \operatorname{cov}\left(\tilde{x}_{t}, \tilde{x}_{t+1}\right)<2 \operatorname{var}\left(\tilde{x}_{t}\right) \text { (by Property II) } \\
\Rightarrow \operatorname{var}\left(\tilde{x}_{t+1}\right)+2 \operatorname{cov}\left(\tilde{x}_{t}, \tilde{x}_{t+1}\right)<\operatorname{var}\left(\tilde{x}_{t}\right) .
\end{gathered}
$$

Since $\operatorname{var}\left(\tilde{x}_{t+1}\right)=\operatorname{var}\left(\tilde{x}_{t}\right)$, this implies thatcov $\left(\tilde{x}_{t}, \tilde{x}_{t+1}\right)<0$,

Hence Property I holds.

b. Property I together with the covariance condition (3) implies Property II.

The proof is by induction.

Let $j=1, \operatorname{var}\left(\tilde{x}_{t}+\tilde{x}_{t+1}\right)=\operatorname{var}\left(\tilde{x}_{t}\right)+\operatorname{var}\left(\tilde{x}_{t+1}\right)+2 \operatorname{cov}\left(\tilde{x}_{t}, \tilde{x}_{t+1}\right)<2 \operatorname{var}\left(\tilde{x}_{t}\right)$

(this follows by Property I and the fact that $\operatorname{var}\left(\tilde{x}_{t}\right)=\operatorname{var}\left(\tilde{x}_{t+1}\right)$ )

Let $j=2, \operatorname{var}\left(\tilde{x}_{t}+\tilde{x}_{t+1}+\tilde{x}_{t+2}\right)$

$=\sum_{j=0}^{2} \operatorname{var}\left(\tilde{x}_{t+j}\right)+2 \operatorname{cov}\left(\tilde{x}_{t}, \tilde{x}_{t+1}\right)+2 \operatorname{cov}\left(\tilde{x}_{t}, \tilde{x}_{t+2}\right)+2 \operatorname{cov}\left(\tilde{x}_{t+1}, \tilde{x}_{t+2}\right)$

\footnotetext{
${ }^{1}$ We thank Awi Federgruen for bringing the FKG inequality to our attention.
} 
By Property I, $2 \operatorname{cov}\left(\tilde{x}_{t}, \tilde{x}_{t+1}\right)<0$

and by condition (3), $2 \operatorname{cov}\left(\tilde{x}_{t}, \tilde{x}_{t+1}\right)+2 \operatorname{cov}\left(\tilde{x}_{t}, \tilde{x}_{t+2}\right)<0$

Again by Property I, $2 \operatorname{cov}\left(\tilde{x}_{t+1}, \tilde{x}_{t+2}\right)<0$. Therefore, $\operatorname{var}\left(\tilde{x}_{t}+\tilde{x}_{t+1}+\tilde{x}_{t+2}\right)<\sum_{j=0}^{2} \operatorname{var}\left(\tilde{x}_{t+j}\right)=3 \operatorname{var}\left(\tilde{x}_{t}\right)$.

Suppose, by Property I and condition $(3), \operatorname{var}\left(\tilde{x}_{t}+\tilde{x}_{t+1}+\ldots+\tilde{x}_{t+j-1}\right)<j \operatorname{var}\left(\tilde{x}_{t}\right)$ holds. We show by induction that this implies

$$
\begin{aligned}
& \operatorname{var}\left(\tilde{x}_{t}+\tilde{x}_{t+1}+\ldots+\tilde{x}_{t+j}\right)<(j+1) \operatorname{var}\left(\tilde{x}_{t}\right) . \\
& \operatorname{var}\left(\tilde{x}_{t}+\tilde{x}_{t+1}+\tilde{x}_{t+2}+\ldots+\tilde{x}_{t+j}\right) \\
& =\operatorname{var}\left(\tilde{x}_{t}+\tilde{x}_{t+1}+\ldots+\tilde{x}_{t+j-1}\right)+\operatorname{var}\left(\tilde{x}_{t+j}\right)+2 \sum_{s=0}^{j-2} \operatorname{cov}\left(\tilde{x}_{t+s}, \tilde{x}_{t+j}\right)+2 \operatorname{cov}\left(\tilde{x}_{t+j-1}, \tilde{x}_{t+j}\right) \\
& <(j) \operatorname{var}\left(\tilde{x}_{t}\right)+\operatorname{var}\left(\tilde{x}_{t+j}\right)+2 \sum_{s=0}^{j-2} \operatorname{cov}\left(\tilde{x}_{t+s}, \tilde{x}_{t+j}\right)+2 \operatorname{cov}\left(\tilde{x}_{t+j-1}, \tilde{x}_{t+j}\right)
\end{aligned}
$$

(by induction)

By Property I, $\operatorname{cov}\left(\tilde{x}_{t+j-1,}, \tilde{x}_{t+j}\right)<0$. Thus by condition (3),

$+2 \sum_{s=0}^{j-2} \operatorname{cov}\left(\tilde{x}_{t+s}, \tilde{x}_{t+j}\right)+2 \operatorname{cov}\left(\tilde{x}_{t+j-1,}, \tilde{x}_{t+j}\right)<0$.

Therefore, since $\operatorname{var}\left(\tilde{x}_{t+j}\right)=\operatorname{var}\left(\tilde{x}_{t}\right)$

$$
\operatorname{var}\left(\tilde{x}_{t}+\tilde{x}_{t+1}+\ldots+\tilde{x}_{t+j}\right)<(j+1) \operatorname{var}\left(\tilde{x}_{t}\right) .
$$

For I $\Rightarrow$ II, the persistence of the series must rapidly decline. 


\section{Proof of Proposition 3.1}

a. The equity price relationship follows from an application of Jensen's inequality:

$$
\begin{aligned}
& \operatorname{cov}\left(\tilde{p}_{t-1}^{e}, \tilde{p}_{t}^{e}\right)=\operatorname{cov}\left(\tilde{k}_{t}, \tilde{k}_{t+1}\right)=\operatorname{cov}\left(\tilde{k}_{t}, \alpha \beta \tilde{k}_{t}^{\alpha} \tilde{\lambda}_{t}\right) \\
& =\alpha \beta E\left(\tilde{\lambda}_{t}\right)\left\{E\left(\tilde{k}_{t}^{1+\alpha}\right)-E\left(\tilde{k}_{t}\right) E\left(\tilde{k}_{t}^{\alpha}\right)\right\} \\
& >\alpha \beta E\left(\tilde{\lambda}_{t}\right)\left\{E\left(\tilde{k}_{t}^{1+\alpha}\right)-E\left(\tilde{k}_{t}\right)\left(E\left(\tilde{k}_{t}\right)\right)^{\alpha}\right\}, \text { since } k_{t}^{\alpha} \text { is concave and } E\left(\tilde{k}_{t}^{\alpha}\right)<\left(E\left(\tilde{k}_{t}\right)\right)^{\alpha}
\end{aligned}
$$

by Jensen's inequality.

$$
=\alpha \beta E\left(\tilde{\lambda}_{t}\right)\left\{E\left(\tilde{k}_{t}^{1+\alpha}\right)-\left(E\left(\tilde{k}_{t}\right)\right)^{1+\alpha}\right\}>0 \text {. }
$$

b. Mean aversion in dividends

$$
\begin{aligned}
& \operatorname{By}(17), \operatorname{cov}\left(\tilde{d}_{t}, \tilde{d}_{t+1}\right)=\operatorname{cov}\left(\alpha(1-\beta) \tilde{k}_{t}^{\alpha} \tilde{\lambda}_{t}, \alpha(1-\beta) \tilde{k}_{t+1}^{\alpha} \tilde{\lambda}_{t+1}\right) \\
& =(\alpha(1-\beta))^{2} \operatorname{cov}\left(\tilde{k}_{t}^{\alpha} \tilde{\lambda}_{t},\left(\alpha \beta \tilde{k}_{t}^{\alpha} \tilde{\lambda}_{t}\right)^{\alpha} \tilde{\lambda}_{t+1}\right) \\
& =(\alpha(1-\beta))^{2}(\alpha \beta)^{\alpha} \operatorname{cov}\left(\tilde{k}_{t}^{\alpha} \tilde{\lambda}_{t}, \tilde{k}_{t}^{\alpha^{2}} \tilde{\lambda}_{t}^{\alpha} \tilde{\lambda}_{t+1}\right) \\
& =(\alpha(1-\beta))^{2}(\alpha \beta)^{\alpha}\left\{E\left(\tilde{k}_{t}^{\alpha+\alpha^{2}} \tilde{\lambda}_{t}^{1+\alpha} \tilde{\lambda}_{t+1}\right)\right. \\
& \left.\quad-E\left(\tilde{k}_{t}^{\alpha} \tilde{\lambda}\right) E\left(\tilde{k}_{t}^{\alpha^{2}} \tilde{\lambda}_{t}^{\alpha} \tilde{\lambda}_{t+1}\right)\right\} \\
& =(\alpha(1-\beta))^{2}(\alpha \beta)^{\alpha} E\left(\tilde{\lambda}_{t+1}\right)\left\{E\left(\tilde{k}_{t}^{\alpha+\alpha^{2}}\right) E\left(\tilde{\lambda}_{t}^{1+\alpha}\right)-E\left(\tilde{k}_{t}^{\alpha}\right) E\left(\tilde{\lambda}_{t}\right) E\left(\tilde{k}_{t}^{\alpha^{2}}\right) E\left(\tilde{\lambda}_{t}^{\alpha}\right)\right\} .
\end{aligned}
$$

By FKG or the Harris inequality

$$
\begin{aligned}
& E\left(\tilde{k}_{t}^{\alpha+\alpha^{2}}\right) \geq E\left(\tilde{k}_{t}^{\alpha}\right) E\left(\tilde{k}_{t}^{\alpha^{2}}\right), \text { and } \\
& E\left(\tilde{\lambda}_{t}^{1+\alpha}\right) \geq E\left(\tilde{\lambda}_{t}\right) E\left(\tilde{\lambda}_{t}^{\alpha}\right) . \text { Thus, }
\end{aligned}
$$




$$
(\alpha(1-\beta))^{2}(\alpha \beta)^{\alpha} E\left(\tilde{\lambda}_{t+1}\right)\left\{E\left(\tilde{k}_{t}^{\alpha+\alpha^{2}}\right) E\left(\tilde{\lambda}_{t}^{1+\alpha}\right)-E\left(\tilde{k}_{t}^{\alpha}\right) E\left(\tilde{\lambda}_{t}\right) E\left(\tilde{k}_{t}^{\alpha^{2}}\right) E\left(\tilde{\lambda}_{t}^{\alpha}\right)\right\} \geq 0 .
$$

c. Derivation of risk free bond price.

$$
\begin{aligned}
p_{t}^{b} & =\beta \int \frac{u_{1}\left(\tilde{c}_{t+1}\right)}{u_{1}\left(c_{t}\right)} d F\left(\tilde{c}_{t+1}, c_{t}\right) \\
& =\beta \int \frac{(1-\alpha \beta) k_{t}^{\alpha} \lambda_{t}}{(1-\alpha \beta)\left[\alpha \beta k_{t}^{\alpha} \lambda_{t}\right]^{\alpha} \tilde{\lambda}_{t+1}} d F\left(\tilde{\lambda}_{t+1}\right) \\
& =\beta \int \frac{1}{(\alpha \beta)^{\alpha}\left[k_{t}^{\alpha} \lambda_{t}\right]^{\alpha-1} \tilde{\lambda}_{t+1}} d F\left(\tilde{\lambda}_{t+1}\right) \\
& =\frac{\beta}{(\alpha \beta)^{\alpha}} k_{t}^{\alpha(1-\alpha)} \lambda_{t}^{1-\alpha} E\left(\lambda_{t+1}^{-1}\right), \text { since }\left\{\tilde{\lambda}_{t}\right\} \text { is i.i.d. }
\end{aligned}
$$

Thus, $p_{t}^{b}=\frac{\beta E\left(\tilde{\lambda}_{t+1}^{-1}\right)}{(\alpha \beta)^{\alpha}} k_{t}^{\alpha(1-\alpha)} \lambda_{t}^{1-\alpha}$, where $E\left(\tilde{\lambda}_{t+1}^{-1}\right)$ is constant for all $t$. As a result, we henceforth omit the time subscript from this term.

$$
\begin{aligned}
& \operatorname{cov}\left(\tilde{p}_{t}^{b}, \tilde{p}_{t+1}^{b}\right)=\operatorname{cov}\left(\left(\frac{\beta E\left(\tilde{\lambda}^{-1}\right)}{(\alpha \beta)^{\alpha}}\right) \tilde{k}_{t}^{\alpha(1-\alpha)} \tilde{\lambda}_{t}^{1-\alpha}, \frac{\beta E\left(\tilde{\lambda}^{-1}\right)}{(\alpha \beta)^{\alpha}} \tilde{k}_{t+1}^{\alpha(1-\alpha)} \tilde{\lambda}_{t+1}^{1-\alpha}\right) \\
& =\left[\frac{\beta E\left(\tilde{\lambda}^{-1}\right)}{(\alpha \beta)^{\alpha}}\right]^{2} \operatorname{cov}\left(\tilde{k}_{t}^{\alpha-\alpha^{2}} \tilde{\lambda}_{t}^{1-\alpha}, \tilde{k}_{t+1}^{\alpha(1-\alpha)} \tilde{\lambda}_{t+1}^{1-\alpha}\right) \\
& =\left[\frac{\beta E\left(\tilde{\lambda}^{-1}\right)}{(\alpha \beta)^{\alpha}}\right]^{2} \operatorname{cov}\left(\tilde{k}_{t}^{\alpha-\alpha^{2}} \tilde{\lambda}_{t}^{1-\alpha},(\alpha \beta)^{\alpha-\sigma^{2}} \tilde{k}_{t}^{\alpha^{2}-\alpha^{3}} \tilde{\lambda}_{t}^{\alpha-\alpha^{2}} \tilde{\lambda}_{t+1}^{1-\alpha}\right), \text { since } k_{t+1}=\alpha \beta k_{t}^{\alpha} \lambda_{t} .
\end{aligned}
$$




$$
=\left[\frac{\beta E\left(\tilde{\lambda}^{-1}\right)}{(\alpha \beta)^{\alpha}}\right]^{2}(\alpha \beta)^{\alpha-\sigma^{2}}\left\{E\left(\tilde{k}_{t}^{\alpha-\alpha^{3}} \tilde{\lambda}_{t}^{1-\alpha^{2}} \tilde{\lambda}_{t+1}^{1-\alpha}\right)-E\left(\tilde{k}_{t}^{\alpha-\alpha^{2}} \tilde{\lambda}_{t}^{1-\alpha}\right) E\left(\tilde{k}_{t}^{\alpha^{2}-\alpha^{3}} \tilde{\lambda}_{t}^{\alpha-\alpha^{2}} \tilde{\lambda}_{t+1}^{1-\alpha}\right)\right\} .
$$

Since $\left\{\lambda_{t}\right\}$ is i.i.d. and the fact that $k_{t}$ is determined in period $t-1$ independent of $\lambda_{t}$ or $\lambda_{t+1}$, we may equivalently write

$$
\begin{aligned}
\operatorname{cov}\left(\tilde{p}_{t}^{b}, \tilde{p}_{t+1}^{b}\right)= & {\left[\frac{\beta E\left(\tilde{\lambda}^{-1}\right)}{(\alpha \beta)^{\alpha}}\right]^{2}(\alpha \beta)^{\alpha-\alpha^{2}}\left\{E\left(\tilde{k}_{t}^{\alpha-\alpha^{3}}\right) E\left(\tilde{\lambda}_{t}^{1-\alpha^{2}}\right) E\left(\tilde{\lambda}_{t+1}^{1-\alpha}\right)\right.} \\
& \left.-E\left(\tilde{k}_{t}^{\alpha-\alpha^{2}}\right) E\left(\tilde{\lambda}_{t}^{1-\alpha}\right) E\left(\tilde{k}_{t}^{\alpha^{2}-\alpha^{3}}\right) E\left(\tilde{\lambda}_{t}^{\alpha-\alpha^{3}}\right) E\left(\tilde{\lambda}_{t+1}^{1-\alpha}\right)\right\} .
\end{aligned}
$$

Let $f_{1}(k)=k^{\alpha-\alpha^{2}}$ and $f_{2}(k)=k^{\alpha^{2}-\alpha^{3}}$.

Since both $f_{1}(k)$ and $f_{2}(k)$ are increasing functions of $k$, and $f_{1}(k) f_{2}(k)=k^{\alpha-\alpha^{3}}$, by FKG or the Harris inequality, $E\left(\tilde{k}_{t}^{\alpha-\alpha^{3}}\right) \geq E\left(\tilde{k}_{t}^{\alpha-\alpha^{2}}\right) E\left(\tilde{k}_{t}^{\alpha^{2}-\alpha^{3}}\right)$.

Similarly,

$E\left(\tilde{\lambda}_{t}^{1-\alpha^{2}}\right) \geq E\left(\tilde{\lambda}_{t}^{1-\alpha}\right) E\left(\tilde{\lambda}_{t}^{\alpha-\alpha^{2}}\right)$

Thus, $\operatorname{cov}\left(\tilde{p}_{t}^{b}, \tilde{p}_{t+1}^{b}\right)=\left[\frac{\beta E\left(\tilde{\lambda}^{-1}\right)}{(\alpha \beta)^{\alpha}}\right]^{2}(\alpha \beta)^{\alpha-\alpha^{2}} E\left(\tilde{\lambda}_{t+1}^{1-\alpha}\right) \geq 0$

The inequality is strict if $\left\{\tilde{\lambda}_{t}\right\}$ is log-normally distributed. The proof in this case follows identically. 


\section{Proof of Proposition 3.2}

a) $\operatorname{cov}\left(\tilde{k}_{t}, \tilde{k}_{t+1}\right)$

$$
\begin{aligned}
& =\operatorname{cov}\left(\tilde{k}_{t}, i\left(\tilde{k}_{t}, \tilde{\lambda}_{t}\right)+(1-\Omega) \tilde{k}_{t}\right) \\
& =\iint\left(\tilde{k}_{t},-\bar{k}\right)\left(i\left(\tilde{k}_{t}, \tilde{\lambda}_{t}\right)+(1-\Omega) \tilde{k}_{t}-\bar{k}\right) d F\left(\tilde{k}_{t}\right) d G\left(\tilde{\lambda}_{t}\right)
\end{aligned}
$$

Let $f_{1}\left(\tilde{k}_{t}, \tilde{\lambda}_{t}\right)=\tilde{k}_{t}-\bar{k}$, and $f_{2}\left(\tilde{k}_{t}, \tilde{\lambda}_{t}\right)=i\left(\tilde{k}_{t}, \tilde{\lambda}_{t}\right)+(1-\Omega) \tilde{k}_{t}-\bar{k}$.

Both $f_{1}()$ and $f_{2}()$ are increasing functions of their arguments by assumption. Hence by FKG or Harris inequality,

$$
\begin{aligned}
& \iint\left(\tilde{k}_{t},-\bar{k}\right)\left(i\left(\tilde{k}_{t}, \tilde{\lambda}_{t}\right)+(1-\Omega) \tilde{k}_{t}-\bar{k}\right) d F\left(\tilde{k}_{t}\right) d G\left(\tilde{\lambda}_{t}\right) \\
& \geq \iint\left(\tilde{k}_{t},-\bar{k}\right) d F\left(\tilde{k}_{t}\right) d G\left(\tilde{\lambda}_{t}\right) \times \iint\left(i\left(\tilde{k}_{t}, \tilde{\lambda}_{t}\right)+(1-\Omega) \tilde{k}_{t}-\bar{k}\right) d F\left(\tilde{k}_{t}\right) d G\left(\tilde{\lambda}_{t}\right)=0
\end{aligned}
$$

Thus, $\operatorname{cov}\left(p_{t-1}^{e}, p_{t}^{e}\right)=\operatorname{cov}\left(\tilde{k}_{t}, \tilde{k}_{t+1}\right) \geq 0$.

In the case of $p_{t}^{b}$,

$$
\begin{aligned}
& \operatorname{cov}\left(p_{t}^{b}, p_{t+1}^{b}\right)=\operatorname{cov}\left(h\left(\tilde{k}_{t}, \tilde{\lambda}_{t}\right), h\left(\tilde{k}_{t+1}, \tilde{\lambda}_{t+1}\right)\right) \text { where } h\left(\tilde{k}_{t}, \lambda_{t}\right) \text { is given by }(10) \\
& =\operatorname{cov}\left(-h\left(\tilde{k}_{t}, \tilde{\lambda}_{t}\right),-h\left(i\left(\tilde{k}_{t}, \tilde{\lambda}_{t}\right)+(1-\Omega) \tilde{k}_{t}, \tilde{\lambda}_{t+1}\right)\right)
\end{aligned}
$$

In general $h\left(\tilde{k}_{t}, \lambda_{t}\right)$ will be a decreasing function of each of its arguments, as it is for specification (15). However, if $h\left(\tilde{k}_{t}, \tilde{\lambda}_{t}\right)$ is a decreasing function $-h\left(\tilde{k}_{t}, \tilde{\lambda}_{t}\right)$ is increasing. Furthermore,

$$
\operatorname{cov}\left(h\left(\tilde{k}_{t}, \tilde{\lambda}_{t}\right), h\left(\tilde{k}_{t+1}, \tilde{\lambda}_{t+1}\right)\right)=\operatorname{cov}\left(-h\left(\tilde{k}_{t}, \tilde{\lambda}_{t}\right),-h\left(\tilde{k}_{t+1}, \tilde{\lambda}_{t+1}\right)\right),
$$

and the argument above may be applied.

\section{Proof of Proposition 3.3}


a) $\quad \operatorname{cov}\left(\tilde{r}_{t}^{e}, \tilde{r}_{t+1}^{e},\right)=\operatorname{cov}\left(\alpha \tilde{k}_{t}^{a-1} \tilde{\lambda}_{t}, \alpha\left[\alpha \beta \tilde{k}_{t}^{\alpha} \tilde{\lambda}_{t}^{\alpha-1} \tilde{\lambda}_{t+1}\right)\right.$

$=\alpha^{2}(\alpha \beta)^{\alpha-1} E\left(\tilde{\lambda}_{t+1}\right)\left\{E\left(\tilde{k}_{t}^{a^{2}-1} \tilde{\lambda}_{t}^{\alpha}\right)-E\left(\tilde{k}_{t}^{\alpha-1} \tilde{\lambda}_{t}\right) E\left(\tilde{k}_{t}^{a^{2}-\alpha} \tilde{\lambda}_{t}^{\alpha-1}\right)\right\}$

(by independence of $\left\{\tilde{\lambda}_{t}\right\}$ ).

$=\alpha^{2}(\alpha \beta)^{\alpha-1} E\left(\tilde{\lambda}_{t+1}\right)\left\{E\left(\tilde{k}_{t}^{a^{2}-1}\right) E\left(\tilde{\lambda}_{t}^{\alpha}\right)-E\left(\tilde{k}_{t}^{\alpha-1}\right) E\left(\tilde{k}_{t}^{a^{2}-\alpha}\right) E\left(\tilde{\lambda}_{t}\right) E\left(\tilde{\lambda}_{t}^{\alpha-1}\right)\right\}$.

We wish first to explore constituents of the preceding expression:

$$
E\left(\tilde{k}_{t}^{a^{2}-1}\right) \text { vs. } E\left(\tilde{k}_{t}^{a-1}\right) E\left(\tilde{k}_{t}^{a^{2}-\alpha}\right) \text {. }
$$

These expressions are of the general form

$E\left(\tilde{k}_{t}^{\gamma_{0}+\gamma_{1}}\right)$ and $E\left(\tilde{k}_{t}^{\gamma_{0}}\right) E\left(\tilde{k}_{t}^{\gamma_{1}}\right)$ where $\gamma_{0}<0, \gamma_{1}<0$.

Define $\tilde{x}_{t}=\tilde{k}_{t}^{\gamma_{0}}$, and $g\left(\tilde{x}_{t}\right)=\tilde{x}_{t}^{\left(\gamma_{1} / \gamma_{0}\right)}$.

Since $\left(\gamma_{1} / \gamma_{0}\right)>0, g\left(\tilde{x}_{t}\right)$ is an increasing function of $\tilde{x}_{t}$, and $g\left(\tilde{x}_{t}\right)=\tilde{k}_{t}^{\gamma_{1}}$.

Thus, $E\left(\tilde{k}_{t}^{\gamma_{0}+\gamma_{1}}\right)=E\left(\tilde{x}_{t} g\left(\tilde{x}_{t}\right)\right)>E\left(\tilde{x}_{t}\right) E\left(g\left(\tilde{x}_{t}\right)\right)=E\left(\tilde{k}_{t}^{\gamma_{0}}\right) E\left(\tilde{k}_{t}^{\gamma_{1}}\right)$

by the FKG or Harris inequality.

Accordingly, $E\left(\tilde{k}_{t}^{\alpha^{2}-1}\right) \geq E\left(\tilde{k}_{t}^{\alpha-1}\right) E\left(\tilde{k}_{t}^{\alpha^{2}-\alpha}\right)$.

We may thus conclude that expression (TA 1) above is

$$
\begin{aligned}
\operatorname{cov}\left(\tilde{r}_{t}^{e}, \tilde{r}_{t+1}^{e},\right) & \geq \alpha^{2}(\alpha \beta)^{\alpha-1} E\left(\tilde{\lambda}_{t+1}\right)\left\{E\left(\tilde{k}_{t}^{a^{2}-1}\right) E\left(\tilde{\lambda}_{t}^{\alpha}\right)-E\left(\tilde{k}_{t}^{a^{2}-1}\right) E\left(\tilde{\lambda}_{t}\right) E\left(\tilde{\lambda}_{t}^{\alpha-1}\right)\right\} \\
& \geq \alpha^{2}(\alpha \beta)^{\alpha-1} E\left(\tilde{\lambda}_{t+1}\right) E\left(\tilde{k}_{t}^{a^{2}-1}\right)\left\{E\left(\tilde{\lambda}_{t}^{\alpha}\right)-E\left(\tilde{\lambda}_{t}\right) E\left(\tilde{\lambda}_{t}^{\alpha-1}\right)\right\} \\
& \geq \alpha^{2}(\alpha \beta)^{\alpha-1} E\left(\tilde{\lambda}_{t+1}\right) E\left(\tilde{k}_{t}^{a^{2}-1}\right)\left\{E\left(\tilde{\lambda}_{t}^{\alpha}\right)-E\left(\tilde{\lambda}_{t}\right)\left(E\left(\tilde{\lambda}_{t}\right)\right)^{\alpha-1}\right\}
\end{aligned}
$$

(by Jensen's inequality, since $\tilde{\lambda}^{\alpha-1}, 0<\alpha<1$ is a convex function of $\tilde{\lambda}$ ) $\operatorname{cov}\left(\tilde{r}_{t}^{e}, \tilde{r}_{t+1}^{e},\right) \geq \alpha^{2}(\alpha \beta)^{\alpha-1} E\left(\tilde{\lambda}_{t+1}\right) E\left(\tilde{k}_{t}^{a^{2}-1}\right)\left\{E\left(\tilde{\lambda}_{t}^{\alpha}\right)-\left(E\left(\tilde{\lambda}_{t}\right)\right)^{\alpha}\right\}$

Again by Jensen's inequality, since $\tilde{\lambda}^{\alpha}, 0<\alpha<1$ is a concave function of $\tilde{\lambda}$ 


$$
E\left(\tilde{\lambda}_{t}^{\alpha}\right)-\left(E\left(\tilde{\lambda}_{t}\right)\right)^{\alpha}<0
$$

Hence, $\operatorname{cov}\left(\tilde{r}_{t}^{e}, \tilde{r}_{t+1}^{e},\right) \geq-M, M>0 \cdot \operatorname{cov}\left(\tilde{r}_{t}^{e}, \tilde{r}_{t+1}^{e},\right)$ can be negative.

b) $\quad \operatorname{cov}\left(\tilde{r}_{t}^{b}, \tilde{r}_{t+1}^{b}\right) \geq 0$.

$$
\begin{aligned}
\operatorname{cov}\left(\tilde{r}_{t}^{b}, \tilde{r}_{t+1}^{b}\right) & =\operatorname{cov}\left(\frac{(\alpha \beta)^{2}}{\beta E\left(\tilde{\lambda}^{-1}\right)} \tilde{k}_{t}^{\alpha(\alpha-1)} \tilde{\lambda}_{t}^{\alpha-1}, \frac{(\alpha \beta)^{2}}{\beta E\left(\tilde{\lambda}^{-1}\right)} \tilde{k}_{t+1}^{\alpha(\alpha-1)} \tilde{\lambda}_{t+1}^{\alpha-1},\right) \\
& =\left(\frac{(\alpha \beta)^{2}}{\beta E\left(\tilde{\lambda}^{-1}\right)}\right)^{2} \operatorname{cov}\left(\tilde{k}_{t}^{\alpha(\alpha-1)} \tilde{\lambda}_{t}^{\alpha-1},\left(\alpha \beta \tilde{k}_{t}^{\alpha} \tilde{\lambda}_{t}^{\alpha(\alpha-1)} \tilde{\lambda}_{t+1}^{\alpha-1}\right)\right. \\
& =(\alpha \beta)^{\alpha(\alpha-1)}\left(\frac{(\alpha \beta)^{2}}{\beta E\left(\tilde{\lambda}^{-1}\right)}\right)^{2} \operatorname{cov}\left(\tilde{k}_{t}^{\alpha^{2}-\alpha} \tilde{\lambda}_{t}^{\alpha-1}, \tilde{k}_{t}^{\alpha^{3}-\alpha^{2}} \tilde{\lambda}_{t}^{\alpha^{2}-\alpha} \tilde{\lambda}_{t+1}^{\alpha-1}\right)
\end{aligned}
$$

By the properties of the covariance function and that $\tilde{k}_{t}, \tilde{\lambda}_{t}$, and $\tilde{\lambda}_{t+1}$ are all independent of one another, the RHS expression becomes $=M E\left(\tilde{\lambda}_{t+1}^{\alpha-1}\right)\left\{E\left(\tilde{k}_{t}^{\alpha^{3}-\alpha}\right) E\left(\tilde{\lambda}_{t}^{\alpha^{2}-1}\right)\right\}-E\left(\tilde{k}_{t}^{\alpha^{2}-\alpha}\right) E\left(\tilde{\lambda}_{t}^{\alpha-1}\right) E\left(\tilde{k}_{t}^{\alpha^{3}-\alpha^{2}}\right) E\left(\tilde{\lambda}_{t}^{\alpha^{2}-\alpha}\right)$

for some positive constant $M$. Let $f_{1}(\tilde{k})=\tilde{k}^{\alpha^{2}-\alpha}$ and $f_{2}(\tilde{k})=\tilde{k}^{\alpha^{3}-\alpha^{2}}$; each is a decreasing function of $k$, furthermore, $f_{1}(\tilde{k}) f_{2}(\tilde{k})=\tilde{k}^{\alpha^{3}-\alpha}$ which is also decreasing in $k$. By the FKG or Harris inequality

$$
\begin{aligned}
& E\left(\tilde{k}_{t}^{\alpha^{3}-\alpha}\right) \geq E\left(\tilde{k}_{t}^{\alpha^{2}-\alpha}\right) E\left(\tilde{k}_{t}^{\alpha^{3}-\alpha^{2}}\right) \text { and } \\
& E\left(\tilde{\lambda}_{t}^{\alpha^{2}-1}\right) \geq E\left(\tilde{\lambda}_{t}^{\alpha-1}\right) E\left(\tilde{\lambda}_{t}^{\alpha^{2}-\alpha}\right) .
\end{aligned}
$$

Thus $\operatorname{cov}\left(\tilde{r}_{t}^{b}, \tilde{r}_{t+1}^{b}\right) \geq 0$.

We have been unable to derive any definitive result for the premium. 


\section{Proof of Proposition 4.1}

This follows simply from the construction of the derived process $\left\{\tilde{\gamma}_{t}^{A B}\right\}$. Every period the process $\left\{\tilde{\gamma}_{t}\right\}$ assumes a value above its mean, it is assuming a value in set $\gamma^{A}$. Furthermore, if the process $\left\{\tilde{\gamma}_{t}^{A B}\right\}$ is in set $\gamma^{A}$, then it is assuming a value above its mean. Thus the average number of periods the process $\left\{\tilde{\gamma}_{t}\right\}$ is above its mean $\left(\tilde{\gamma}_{t} \in \gamma^{A}\right)$ must coincide with the average number of periods it is in set $\gamma^{A}$. Thus $A C T_{\left\{\tilde{\gamma}_{t}\right\}}^{A}=A C T_{\left\{\tilde{\gamma}_{t}^{A B}\right\}}^{A}$. A similar identification establishes that $A C T_{\left\{\tilde{\gamma}_{t}\right\}}^{B}=A C T_{\left\{\tilde{\gamma}_{t}^{A B}\right\}}^{B}$.

Properties of $\left\{\tilde{\gamma}^{A B}\right\}$

We first restrict attention to a consideration of arbitrary two state Markov chains. We adopt the convention that any measurement of the "time to crossing' includes the crossing period itself.

A. Consider an arbitrary two state Markov chain with states $\gamma_{1}, \gamma_{2}$ and transition matrix:

$$
\gamma_{1}\left[\begin{array}{cc}
\gamma_{1} & \gamma_{2} \\
\varphi_{1} & 1-\varphi_{1} \\
1-\varphi_{2} & \varphi_{2}
\end{array}\right]
$$

where $0<\varphi_{1}<1$ and $0<\varphi_{2}<1$. The associated ergodic probability distribution $\left(\pi_{1}, \pi_{2}\right)$ satisfies

$$
\left[\pi_{1}, \pi_{2}\right]=\left[\pi_{1}, \pi_{2}\right]\left[\begin{array}{cc}
\varphi_{1} & 1-\varphi_{1} \\
1-\varphi_{2} & \varphi_{2}
\end{array}\right]
$$


with solution $\pi_{1}=\frac{1-\phi_{2}}{2-\left(\phi_{1}+\phi_{2}\right)}$ and $\pi_{2}=\frac{1-\phi_{1}}{2-\left(\phi_{1}+\phi_{2}\right)}$

B. Suppose the process is in state $\tilde{\gamma}=\gamma_{1}$. The average time to crossing to state $2, A C T_{1}$, is given by:

$$
\begin{aligned}
& A C T_{1}=\sum_{n=1}^{\infty} n \operatorname{Prob}\left(\begin{array}{c}
\tilde{\gamma}=\gamma_{2} \\
\text { in step } n \quad \text { the } n-1 \text { prior steps }
\end{array}\right) \\
& =\sum_{n=1}^{\infty} n \varphi_{1}^{n-1}\left(1-\varphi_{1}\right)=\left(1-\varphi_{1}\right) \sum_{n=1}^{\infty} n \varphi_{1}^{n}=\left(1-\varphi_{1}\right)\left(\frac{1}{\left(1-\varphi_{1}\right)^{2}}\right)=\frac{1}{1-\varphi_{1}}
\end{aligned}
$$

Similarly, $A C T_{2}=\frac{1}{1-\varphi_{2}}$

Accordingly, the average crossing time, $A C T$, satisfies

$$
\begin{aligned}
& \qquad A C T=\pi_{1} A C T_{1}+\pi_{2} A C T_{2}=\pi_{1}\left(\frac{1}{1-\varphi_{1}}\right)+\pi_{2}\left(\frac{1}{1-\varphi_{2}}\right) \\
& =\frac{1}{2-\left(\phi_{1}+\phi_{2}\right)}\left[\frac{1-\phi_{2}}{1-\phi_{1}}+\frac{1-\phi_{1}}{1-\phi_{2}}\right] \\
& \text { C. We compute } \operatorname{corr}\left(\tilde{\gamma}_{t}, \tilde{\gamma}_{t+1}\right)=\frac{\operatorname{cov}\left(\tilde{\gamma}_{t}, \tilde{\gamma}_{t+1}\right)}{\sigma_{\tilde{\gamma}_{t}} \sigma_{\tilde{\gamma}_{t+1}}} \\
& =\frac{E\left(\tilde{\gamma}_{t} \tilde{\gamma}_{t+1}\right)-E\left(\tilde{\gamma}_{t}\right) E\left(\tilde{\gamma}_{t+1}\right)}{\sigma_{\tilde{\gamma}_{t}} \sigma_{\tilde{\gamma}_{t+1}}}
\end{aligned}
$$

Without loss of generality, we assume $\gamma_{1}=1$ and $\gamma_{2}=-1$, since the $\operatorname{corr}\left(\tilde{\gamma}_{t}, \tilde{\gamma}_{t+1}\right)$ is determined by the structure of the transition matrix and not the specific values assumed by $\tilde{\gamma}_{t} \cdot{ }^{2}$

\footnotetext{
${ }^{2}$ Consider a stochastic process $\tilde{\gamma}_{t} ;$ then for any $a>0$ and any $b \in \mathbb{R}, \operatorname{corr}\left(\tilde{\gamma}_{t}, \tilde{\gamma}_{t+1}\right)=\operatorname{corr}\left(a \tilde{\gamma}_{t}+b, a \tilde{\gamma}_{t+1}+b\right)$.
} 
The correlation computation requires the following constituents:

(i) $E\left(\tilde{\gamma}_{t}\right)=\gamma_{1}\left(\frac{1-\phi_{2}}{2-\left(\phi_{1}+\phi_{2}\right)}\right)+\gamma_{2}\left(\frac{1-\phi_{1}}{2-\left(\phi_{1}+\phi_{2}\right)}\right)$

$$
=\frac{\phi_{1}-\phi_{2}}{2-\left(\phi_{1}+\phi_{2}\right)}
$$

(ii) $E\left(\tilde{\gamma}_{t+1}\right)=\left(\frac{\phi_{1}-\phi_{2}}{2-\left(\phi_{1}+\phi_{2}\right)}\right)$

(iii) $\operatorname{var}\left(\tilde{\gamma}_{t}\right)=E\left(\tilde{\gamma}_{t}-E\left(\tilde{\gamma}_{t}\right)\right)^{2}$

$$
\begin{aligned}
= & \left(\frac{1-\phi_{2}}{2-\left(\phi_{1}+\phi_{2}\right)}\right)\left(1-\frac{\phi_{1}-\phi_{2}}{2-\left(\phi_{1}+\phi_{2}\right)}\right)^{2} \\
& \quad+\left(\frac{1-\phi_{1}}{2-\left(\phi_{1}+\phi_{2}\right)}\right)\left(-1-\frac{\phi_{1}-\phi_{2}}{2-\left(\phi_{1}+\phi_{2}\right)}\right)^{2} \\
= & \left(\frac{1}{2-\left(\phi_{1}+\phi_{2}\right)}\right)^{3}\left[\left(1-\phi_{2}\right)\left(2-2 \phi_{1}\right)^{2}+\left(1-\phi_{1}\right)\left(-2+2 \phi_{2}\right)^{2}\right] \\
= & \frac{4\left(1-\phi_{1}\right)\left(1-\phi_{2}\right)}{\left[2-\left(\phi_{1}+\phi_{2}\right)\right]^{3}}\left\{2-\left(\phi_{1}+\phi_{2}\right)\right\} \\
= & \frac{4\left(1-\phi_{1}\right)\left(1-\phi_{2}\right)}{\left[2-\left(\phi_{1}+\phi_{2}\right)\right]^{2}}=\operatorname{var}\left(\tilde{\gamma}_{t+1}\right)
\end{aligned}
$$

We can thus assume $\gamma_{1}=1$ and $\gamma_{2}=-1$, if constants $a, b$ as per the above correlation equality satisfy $a \gamma_{1}+b=1$ and $a \gamma_{2}+b=-1$. The solution to this simple system of equations is $a=\frac{2}{\gamma_{1}-\gamma_{1}}$ and $b=\left(-1+\gamma_{2} / \gamma_{1}\right) /\left(1-\gamma_{2} / \gamma_{1}\right)$. 
(iv) $E\left(\tilde{\gamma}_{t} \tilde{\gamma}_{t+1}\right)=\sum_{\gamma_{t}} \operatorname{Prob}\left(\gamma_{t}\right)\left[\sum_{\gamma_{t+1}} \gamma_{t+1} \operatorname{Prob}\left(\gamma_{t+1} \mid \gamma_{t}\right)\right]\left(\gamma_{t}\right)$

$$
\begin{aligned}
= & 1\left[\frac{1-\phi_{2}}{2-\left(\phi_{1}+\phi_{2}\right)}\right]\left[1 \phi_{1}+\left(1-\phi_{1}\right)(-1)\right] \\
& \quad+(-1)\left[\frac{1-\phi_{1}}{2-\left(\phi_{1}+\phi_{2}\right)}\right]\left[1\left(1-\phi_{2}\right)\left(-1 \phi_{2}\right)\right] \\
= & \frac{1}{2-\left(\phi_{1}+\phi_{2}\right)}\left\{\left(1-\phi_{2}\right)\left[-1+2 \phi_{1}\right]-\left(1-\phi_{1}\right)\left[1-2 \phi_{2}\right]\right\} \\
= & \frac{1}{2-\left(\phi_{1}+\phi_{2}\right)}\left\{-2+3 \phi_{1}+3 \phi_{2}-4 \phi_{1} \phi_{2}\right\}
\end{aligned}
$$

(v) $\operatorname{corr}\left(\tilde{\gamma}_{t}, \tilde{\gamma}_{t+1}\right)=\frac{E\left(\tilde{\gamma}_{t} \tilde{\gamma}_{t+1}\right)-E\left(\tilde{\gamma}_{1}\right) E\left(\tilde{\gamma}_{t+1}\right)}{\sigma_{\tilde{\gamma}_{t}} \sigma_{\tilde{\gamma}_{t+1}}}$

$$
\begin{gathered}
=\frac{\frac{1}{2-\left(\phi_{1}+\phi_{2}\right)}\left[-2+3\left(\phi_{1}+\phi_{2}\right)-4 \phi_{1} \phi_{2}\right]-\left[\frac{\phi_{1}-\phi_{2}}{2-\left(\phi_{1}+\phi_{2}\right)}\right]^{2}}{\frac{4\left(1-\phi_{1}\right)\left(1-\phi_{2}\right)}{\left(2-\left(\phi_{1}+\phi_{2}\right)\right)^{2}}} \\
=\frac{\left[2-\left(\phi_{1}+\phi_{2}\right)\right]\left[-2+3\left(\phi_{1}+\phi_{2}\right)-4 \phi_{1} \phi_{2}\right]-\left[\phi_{1}-\phi_{2}\right]^{2}}{4\left(1-\phi_{1}\right)\left(1-\phi_{2}\right)}
\end{gathered}
$$

(after considerable simplification)

$$
\begin{aligned}
& =\frac{-4+\left(8+4 \phi_{1} \phi_{2}\right)\left[\phi_{1}+\phi_{2}\right]-12 \phi_{1} \phi_{2}-4 \phi_{1}^{2}-4 \phi_{2}^{2}}{4\left(1-\phi_{1}\right)\left(1-\phi_{2}\right)} \\
& =\frac{-\left(1+\phi_{1} \phi_{2}\right)+\left(\phi_{1}+\phi_{2}\right)\left[1+\left(1-\phi_{1}\right)\left(1-\phi_{2}\right)\right]}{\left(1-\phi_{1}\right)\left(1-\phi_{2}\right)} \\
& =\left(\phi_{1}+\phi_{2}\right)+\left[\frac{-\left(1-\phi_{1}\right)\left(1-\phi_{2}\right)}{\left(1-\phi_{1}\right)\left(1-\phi_{2}\right)}\right]=\left(\phi_{1}+\phi_{2}\right)-1
\end{aligned}
$$


D. Proposition 4.2. To show $A C T_{\left\{\tilde{\gamma}_{t}\right\}} \leq 2$ implies $\operatorname{corr}\left(\tilde{\gamma}_{t}, \tilde{\gamma}_{t+1}\right) \leq 0$.

We show $A C T \leq 2$ implies $\left(\phi_{1}+\phi_{2}\right) \leq 1$.

$$
\begin{aligned}
A C T_{\left\{\tilde{\gamma}_{t}\right\}} \leq 2 & \Rightarrow \\
& \frac{1}{2-\left(\phi_{1}+\phi_{2}\right)}\left[\frac{1-\phi_{1}}{1-\phi_{2}}+\frac{1-\phi_{2}}{1-\phi_{1}}\right] \leq 2
\end{aligned}
$$

The second term in the $A C T$ expression is of the form $x+\frac{1}{x}$, which assumes a minimum at $x=1$. Therefore,

$$
\begin{gathered}
\frac{1}{2-\left(\phi_{1}+\phi_{2}\right)}[2] \leq A C T_{\left\{\tilde{\gamma}_{t}\right\}} \leq 2, \text { or } \\
\frac{2}{2-\left(\phi_{1}+\phi_{2}\right)} \leq 2 ; \text { equivalently } \\
1 \leq 2-\left(\phi_{1}+\phi_{2}\right) \text { or } \\
\left(\phi_{1}+\phi_{2}\right) \leq 1 .
\end{gathered}
$$

Suppose $\phi_{1}=\phi_{2}$ and $\left(\phi_{1}+\phi_{2}\right) \leq 1$.

Then $A C T_{\left\{\tilde{\gamma}_{t}\right\}}=\frac{1}{2-\left(\phi_{1}+\phi_{2}\right)}\left[\frac{1-\phi_{1}}{1-\phi_{2}}+\frac{1-\phi_{2}}{1-\phi_{1}}\right] \leq\left[\frac{1-\phi_{1}}{1-\phi_{2}}+\frac{1-\phi_{2}}{1-\phi_{1}}\right]=2$

E. When $\phi_{1}=\phi_{2}=\phi$, the $A C T$ reduces to

$$
\begin{aligned}
& A C T=\frac{1}{2-2 \phi}[1+1]=\frac{1}{1-\phi}, \text { and } \\
& \operatorname{corr}\left(\tilde{\gamma}_{t}, \tilde{\gamma}_{t+1}\right)=2 \phi-1 . \text { Thus }
\end{aligned}
$$




$$
\begin{aligned}
& \operatorname{corr}\left(\gamma_{t}^{x}, \gamma_{t+1}^{x}\right) \geq \operatorname{corr}\left(\gamma_{t}^{y}, \gamma_{t+1}^{y}\right), \text { if and only if } \\
& 2 \phi^{x}-1>2 \phi^{y}-1, \text { if and only if } \\
& \phi^{x}>\phi^{y}, \text { if and only if } \\
& \frac{1}{1-\phi^{x}}>\frac{1}{1-\phi^{y}}, \text { if and only if } \\
& A C T_{\left\{\gamma_{t}^{x}\right\}}>A C T_{\left\{\gamma_{t}^{y}\right\}} .
\end{aligned}
$$

F. The region A is computed by searching for the pairs $(0,0)<\left(\phi_{1}, \phi_{2}\right)<(1,1)$ such that

$$
\partial A C T / \partial \phi_{1}>0 \text { and } \partial A C T / \partial \phi_{2}>0 \text {. }
$$

The indicated region (Figure 2) was constructed numerically.

\section{Proof of Proposition 5.1}

(a) We first offer the proof for $\left\{\tilde{p}_{t}^{e}\right\} ;\left\{\tilde{d}_{t}\right\}$ is analyzed similarly, since

$$
\begin{aligned}
& d_{t}=((1-\beta) / \beta) p_{t}^{e} \\
& \operatorname{cov}\left(\tilde{p}_{t}^{e}, \tilde{p}_{t+1}^{e}\right)= \operatorname{cov}\left(\tilde{k}_{t+1}, \tilde{k}_{t+2}\right) \\
&=\operatorname{cov}\left(\alpha \beta \tilde{k}_{t}^{\alpha} e^{\tilde{\lambda}_{t}}, \alpha \beta\left[\alpha \beta \tilde{k}_{t}^{\alpha} e^{\tilde{\lambda}_{t}}\right]^{\alpha} e^{\tilde{\lambda}_{t+1}}\right) \\
&=\operatorname{cov}\left(\alpha \beta \tilde{k}_{t}^{\alpha} e^{\tilde{\lambda}_{t}},(\alpha \beta)^{1+\alpha} \tilde{k}_{t}^{\alpha^{2}} e^{\alpha \tilde{\lambda}_{t}} e^{\left(\rho \tilde{\lambda}_{t}+\tilde{\varepsilon}_{t+1}\right)}\right) \\
&=(\alpha \beta)^{2+\alpha} \operatorname{cov}\left(\tilde{k}_{t}^{\alpha} e^{\tilde{\lambda}_{t}}, \tilde{k}_{t}^{\alpha^{2}} e^{(\alpha+\rho) \tilde{\lambda}_{t}} \cdot e^{\tilde{\varepsilon}_{t+1}}\right) \\
&=(\alpha \beta)^{2+\alpha} E\left(e^{\tilde{\varepsilon}_{t+1}}\right)\left\{E\left(\tilde{k}_{t}^{\alpha+\alpha^{2}} e^{(\alpha+\rho+1) \tilde{x}_{t}}\right)-E\left(\tilde{k}_{t}^{\alpha} e^{\tilde{\lambda}_{t}}\right) E\left(\tilde{k}_{t}^{\alpha^{2}} e^{(\alpha+\rho) \tilde{\lambda}_{t}}\right)\right\} .
\end{aligned}
$$

Let $g^{1}\left(k_{t}, \lambda_{t}\right)=\tilde{k}_{t}^{\alpha} e^{\tilde{\lambda}_{t}}, g_{1}^{1}>0 ; g_{2}^{1}>0$ 
$g^{2}\left(k_{t}, \lambda_{t}\right)=\tilde{k}_{t}^{\alpha^{2}} e^{(\alpha+\rho) \tilde{\lambda}_{t}}, g_{1}^{2}>0 ; g_{2}^{2}>0$

By FKG or the Harris inequality

$$
\begin{aligned}
& E\left(g^{1}\left(\tilde{k}_{t}, \tilde{\lambda}_{t}\right) g^{2}\left(\tilde{k}_{t}, \tilde{\lambda}_{t}\right)\right) \\
& \quad \geq E\left(g^{1}\left(\tilde{k}_{t}, \tilde{\lambda}_{t}\right)\right) E\left(g^{2}\left(\tilde{k}_{t}, \tilde{\lambda}_{t}\right)\right)
\end{aligned}
$$

Thus, $\operatorname{cov}\left(\tilde{p}_{t}^{e}, \tilde{p}_{t+1}^{e}\right)=\operatorname{cov}\left(\tilde{k}_{t+1}^{e}, \tilde{k}_{t+2}^{e}\right) \geq 0$.

(b) $\operatorname{cov}\left(\tilde{p}_{t}^{b}, \tilde{p}_{t+1}^{b}\right)$

where $p_{t}^{b}=\beta e^{\sigma_{\varepsilon}^{2} / 2}(\alpha \beta)^{-2} k_{t}^{\alpha-\alpha^{2}} e^{(1-\alpha-\rho) \tilde{\lambda}_{t}}$

$$
\begin{aligned}
& \operatorname{cov}\left(\tilde{p}_{t}^{b}, \tilde{p}_{t+1}^{b}\right)=\beta^{2} e^{\sigma_{\varepsilon}^{2}}(\alpha \beta)^{-2 \alpha} \operatorname{cov}\left(\tilde{k}_{t}^{\alpha-\alpha^{2}} e^{(1-\alpha-\rho) \tilde{x}_{t}},\left(\alpha \beta \tilde{k}_{t}^{\alpha} e^{\tilde{x}_{t}}\right)^{\alpha-\alpha^{2}} e^{(1-\alpha-\rho)\left(\rho \tilde{x}_{t}+\tilde{\varepsilon}_{t+1}\right)}\right) \\
& =\beta^{2} e^{\sigma_{\varepsilon}^{2}}(\alpha \beta)^{-2 \alpha}(\alpha \beta)^{\alpha-\alpha^{2}} \operatorname{cov}\left(\tilde{k}_{t}^{\alpha-\alpha^{2}} e^{(1-\alpha-\rho) \tilde{x}_{t}}, \tilde{k}_{t}^{\alpha^{2}-\alpha^{3}} e^{\left(\alpha-\alpha^{2}\right) \tilde{x}_{t}} e^{(1-\alpha-\rho)\left(\rho \tilde{\lambda}_{t}+\tilde{\varepsilon}_{t+1}\right)}\right) \\
& \mathrm{M} \\
& =\mathrm{M}\left(\operatorname{cov} \tilde{k}_{t}^{\alpha-\alpha^{2}} e^{(1-\alpha-\rho) \tilde{t}_{t}}, \tilde{k}_{t}^{\alpha^{2}-\alpha^{3}} e^{\left(\alpha-\alpha^{2}+\rho(1-\alpha-\rho)\right) \tilde{x}_{t}} e^{(1-\alpha-\rho) \tilde{t}_{t+1}}\right) \\
& =M e^{(1-\alpha-\rho)^{2} \sigma_{\varepsilon}^{2} / 2}\left\{E\left(\tilde{k}_{t}^{\alpha-\alpha^{3}} e^{\left(\alpha-\alpha^{2}+(1+\rho)(1-\alpha-\rho) \tilde{t}_{t}\right)}\right)-E\left(\tilde{k}_{t}^{\alpha-\alpha^{2}} e^{(1-\alpha-\rho) \tilde{x}_{t}}\right) E\left(\tilde{k}_{t}^{\alpha^{2}-\alpha^{3}} e^{\left(\alpha-\alpha^{2}+\rho(1-\alpha-\rho)\right) \tilde{x}_{t}}\right)\right\}
\end{aligned}
$$

If we let $g\left(\tilde{k}_{t}, \tilde{\lambda}_{t}\right)=\tilde{k}_{t}^{\alpha-\alpha^{2}} e^{(1-\alpha-\rho) \tilde{x}_{t}}$

and

$f\left(\tilde{k}_{t}, \tilde{\lambda}_{t}\right)=\tilde{k}_{t}^{\alpha^{2}-\alpha^{3}} e^{\left(\alpha-\alpha^{2}+\rho(1-\alpha-\rho)\right) \tilde{\lambda}_{t}}$,

then, if $\alpha+\rho<1$,

$$
f_{1}(\quad)>0 \quad f_{2}(\quad)>0, g_{1}(\quad)>0 \quad g_{2}(\quad)>0 .
$$


By the FKG or the Harris inequality we conclude immediately that $\operatorname{cov}\left(\tilde{p}_{t}^{b}, \tilde{p}_{t+1}^{b}\right) \geq 0$, provided $\alpha+\rho<1$.

\section{Proof of Proposition 5.2}

(a) $\operatorname{cov}\left(\tilde{r}_{t}^{b}, \tilde{r}_{t+1}^{b}\right)=\operatorname{cov}\left(\tilde{r}_{t+1}^{b}, \tilde{r}_{t+2}^{b}\right)$

$$
=\underbrace{\left[\frac{(\alpha \beta)^{\alpha}}{\beta} e^{-\sigma_{\varepsilon}^{2} / 2}\right]^{2}}_{L} \operatorname{cov}\left(\tilde{k}_{t}^{\alpha^{2}-\alpha} e^{(\alpha+\rho-1) \tilde{\lambda}_{t}}, \tilde{k}_{t+1}^{\alpha^{2}-\alpha} e^{(\alpha+\rho-1) \tilde{\lambda}_{t+1}}\right)
$$

But $k_{t+1}=\alpha \beta k_{t}^{\alpha} e^{\tilde{\lambda}_{t}}$ and $\tilde{\lambda}_{t+1}=\rho \tilde{\lambda}_{t}+\tilde{\varepsilon}_{t+1}$; thus:

$$
\begin{aligned}
& =L(\alpha \beta)^{\alpha^{2}-\alpha} \operatorname{cov}\left(\tilde{k}_{t}^{\alpha^{2}-\alpha} e^{(\alpha+\rho-1) \tilde{\lambda}_{t}}, \tilde{k}_{t}^{\alpha^{3}-\alpha^{2}} e^{\tilde{\lambda}_{t}\left(\alpha^{2}-\alpha\right)} e^{(\alpha+\rho-1) \rho \tilde{\lambda}_{t}+(\alpha+\rho-1) \tilde{\varepsilon}_{t+1}}\right) \\
& =L(\alpha \beta)^{\alpha^{2}-\alpha} \operatorname{cov}\left(\tilde{k}_{t}^{\alpha^{2}-\alpha} e^{(\alpha+\rho-1) \tilde{x}_{t}}, \tilde{k}_{t}^{\alpha^{3}-\alpha^{2}} e^{\left[\left(\alpha^{2}-\alpha\right)+(\alpha+\rho-1) \rho\right) \tilde{\lambda}_{t}} \cdot e^{(\alpha+\rho-1) \tilde{\varepsilon}_{t+1}}\right)
\end{aligned}
$$$$
=L(\alpha \beta)^{\alpha^{2}-\alpha} e^{(\alpha+\rho-1)^{2} \sigma_{\varepsilon}^{2} / 2}\left\{E\left(\tilde{k}_{t}^{\alpha^{3}-\alpha} e^{\left[\left(\alpha^{2}-\alpha\right)+2(\alpha+\rho-1)\right] \tilde{\lambda}_{t}}\right)-E\left(\tilde{k}_{t}^{\alpha^{2}-\alpha} e^{(\alpha+\rho-1) \tilde{t}_{t}}\right) E\left(\tilde{k}_{t}^{\alpha^{3}-\alpha^{2}} e^{\left[\left(\alpha^{2}-\alpha\right)+(\alpha+\rho-1) \rho\right] \tilde{\lambda}_{t}}\right)\right\}
$$

Let $g^{1}(\tilde{k}, \tilde{\lambda})=\left(\tilde{k}_{t}^{\alpha^{2}-\alpha}\right) e^{(\alpha+\rho-1) \tilde{x}_{t}}$

$g^{2}(\tilde{k}, \tilde{\lambda})=\tilde{k}_{t}^{\alpha^{3}-\alpha^{2}} e^{\left[\left(\alpha^{2}-\alpha\right)+(\alpha+\rho-1) \rho\right) \tilde{\lambda}_{t}}$

$g_{1}^{1}(\tilde{k}, \tilde{\lambda})<0 \quad g_{2}^{1}(\tilde{k}, \tilde{\lambda})<0 \quad$ if $\alpha+\rho<1$

$g_{1}^{2}(\tilde{k}, \tilde{\lambda})<0 \quad g_{2}^{2}(\tilde{k}, \tilde{\lambda})<0 \quad$ if $\alpha+\rho<1$.

Hence, $-g^{1}\left(\tilde{k}_{t}, \tilde{\lambda}_{t}\right)$ is increasing

and $-g^{2}\left(\tilde{k}_{t}, \tilde{\lambda}_{t}\right)$ is increasing. Thus,

$$
\int-g^{1}\left(\tilde{k}_{t}, \tilde{\lambda}_{t}\right)\left(-g^{2}\left(\tilde{k}_{t}, \tilde{\lambda}_{t}\right)\right) d F\left(\tilde{k}_{t}, \tilde{\lambda}_{t}\right) \geq \int-g^{1}\left(\tilde{k}_{t}, \tilde{\lambda}_{t}\right) d F\left(\tilde{k}_{t}, \tilde{\lambda}_{t}\right) \int-g^{2}\left(\tilde{k}_{t}, \tilde{\lambda}_{t}\right) d F\left(\tilde{k}_{t}, \tilde{\lambda}_{t}\right)
$$




$$
\int g^{1}\left(\tilde{k}_{t}, \tilde{\lambda}_{t}\right) g^{2}\left(\tilde{k}_{t}, \tilde{\lambda}_{t}\right) d F\left(\tilde{k}_{t}, \tilde{\lambda}_{t}\right) \geq \int g^{1}\left(\tilde{k}_{t}, \tilde{\lambda}_{t}\right) d F\left(\tilde{k}_{t}, \tilde{\lambda}_{t}\right) \int g^{2}\left(\tilde{k}_{t}, \tilde{x}_{t}\right) d F\left(\tilde{k}_{t}, \tilde{\lambda}_{t}\right)
$$

(by the FKG or the Harris inequality).

Thus, $\operatorname{cov}\left(\tilde{r}_{t}^{b}, \tilde{r}_{t+1}^{b}\right) \geq 0$.

(b) $\operatorname{cov}\left(\tilde{r}_{t}^{e}, \tilde{r}_{t+1}^{e}\right)$

$$
\begin{aligned}
= & \operatorname{cov}\left(\alpha \tilde{k}_{t}^{\alpha-1} e^{\tilde{\lambda}_{t}}, \alpha \tilde{k}_{t+1}^{\alpha-1} e^{\tilde{\lambda}_{t+1}}\right) \\
= & \operatorname{cov}\left(\alpha \tilde{k}_{t}^{\alpha-1} e^{\tilde{\lambda}_{t}}, \alpha\left(\alpha \beta \tilde{k}_{t}^{\alpha} e^{\tilde{\lambda}_{t}}\right)^{\alpha-1} e^{\rho \tilde{\lambda}_{t}+\tilde{\varepsilon}_{t+1}}\right) \\
= & \operatorname{cov}\left(\alpha \tilde{k}_{t}^{\alpha-1} e^{\tilde{\lambda}_{t}}, \alpha^{\alpha} \beta^{\alpha-1} \tilde{k}_{t}^{\alpha^{2-\alpha}} e^{(\alpha-1) \tilde{x}_{t}} e^{\rho \tilde{\lambda}_{t}+\tilde{\varepsilon}_{t+1}}\right) \\
= & \underbrace{1+\alpha} \beta^{\alpha-1} \operatorname{cov}\left(\tilde{k}_{t}^{\alpha-1} e^{\tilde{\lambda}_{t}}, \tilde{k}_{t}^{\alpha^{2-\alpha}} e^{(\alpha+\rho-1) \tilde{\lambda}_{t}} e^{\tilde{\varepsilon}_{t+1}}\right) \\
= & M\left\{E\left(\tilde{k}_{t}^{\alpha^{2}-1} e^{(\alpha+\rho) \tilde{x}_{t}} e^{\tilde{\varepsilon}_{t+1}}\right)-E\left(\tilde{k}_{t}^{\alpha-1} e^{\tilde{\lambda}_{t}}\right) E\left(\tilde{k}_{t}^{\alpha^{2}-\alpha} e^{(\alpha+\rho-1) \tilde{\lambda}_{t}} e^{\tilde{\varepsilon}_{t+1}}\right)\right\} \\
= & M e^{\sigma_{\varepsilon}^{2} / 2}\left\{E\left(\tilde{k}_{t}^{\alpha^{2}-1} e^{(\alpha+\rho) \tilde{x}_{t}}\right)-E\left(\tilde{k}_{t}^{\alpha-1} e^{\tilde{t}_{t}}\right) E\left(\tilde{k}_{t}^{\alpha^{2}-\alpha} e^{(\alpha+\rho-1) \tilde{x}_{t}}\right)\right\}
\end{aligned}
$$

(i) From the proof of Proposition 3.3,

$$
E\left(\tilde{k}_{t}^{\alpha^{2}-1}\right)>E\left(\tilde{k}_{t}^{\alpha-1}\right) E\left(\tilde{k}_{t}^{\alpha^{2}-\alpha}\right) .
$$

(ii) If $(\alpha+\rho)>1$, then $e^{(\alpha+\rho) \tilde{\lambda}_{t}}, e^{\tilde{\lambda}_{t}}$ and $e^{(\alpha+\rho-1) \tilde{\tau}_{t}}$ are all increasing functions of $\tilde{\lambda}_{t}$. Thus, by the FKG or the Harris inequality,

$$
E\left(e^{(\alpha+\rho) \tilde{\lambda}_{t}}\right) \geq E\left(e^{\tilde{t}_{t}}\right) E\left(e^{(\alpha+\rho-1) \tilde{\lambda}_{t}}\right) .
$$

Since (TA 2) is equivalent to (TA 3)

$$
=M e^{\sigma_{\varepsilon}^{2} / 2}\left\{E\left(\tilde{k}_{t}^{\alpha^{2}-1}\right) E\left(e^{(\alpha+\rho) \tilde{x}_{t}}\right)-E\left(\tilde{k}_{t}^{\alpha-1}\right) E\left(e^{\tilde{\lambda}_{t}}\right) E\left(\tilde{k}_{t}^{\alpha^{2}-\alpha}\right) E\left(e^{(\alpha+\rho-1) \tilde{x}_{t}}\right)\right\}
$$


then by relationship (i), (ii) noted previously, we have $\operatorname{cov}\left(\tilde{r}_{t}^{e}, \tilde{r}_{t+1}^{e}\right) \geq 0$, provided $(\alpha+\rho)>1$.

Proof of Proposition 5.3 (This result is due to Sergio Villar)

This proof uses the fact that if $\tilde{y} \sim N(0, v)$, then $E[\exp (\tilde{y})]=\frac{v}{2}$

For the $A R(1)$ process, $\operatorname{Var}\left(\tilde{x}_{t}\right)=\sigma^{2}\left(\frac{1-\rho^{2 t}}{1-\rho^{2}}\right)$ :

$$
\begin{aligned}
& \operatorname{Cov}\left(\tilde{\lambda}_{t}, \tilde{\lambda}_{t+1}\right)=\operatorname{Cov}\left(\exp \left(\tilde{x}_{t}\right), \exp \left(\rho \tilde{x}_{t}+\tilde{\varepsilon}_{t+1}\right)\right) \\
& =E\left[\exp \left(\tilde{x}_{t}\right) \exp \left(\rho \tilde{x}_{t}+\tilde{\varepsilon}_{t+1}\right)\right]-E\left[\exp \left(\tilde{x}_{t}\right)\right] E\left[\exp \left(\rho \tilde{x}_{t}+\tilde{\varepsilon}_{t+1}\right)\right] \\
& =E\left[\exp \left((\rho+1) \tilde{x}_{t}+\tilde{\varepsilon}_{t+1}\right)\right]-E\left[\exp \left(\tilde{x}_{t}\right)\right] E\left[\exp \left(\rho \tilde{x}_{t}+\tilde{\varepsilon}_{t+1}\right)\right] \\
& =E\left[\exp \left((\rho+1) \tilde{x}_{t}\right)\right] E\left[\exp \left(\tilde{\varepsilon}_{t+1}\right)\right]-E\left[\exp \left(\tilde{x}_{t}\right)\right] E\left[\exp \left(\rho \tilde{x}_{t}\right)\right] E\left[\exp \left(\tilde{\varepsilon}_{t+1}\right)\right] \\
& =E\left[\exp \left(\tilde{\varepsilon}_{t+1}\right)\right]\left(E\left[\exp \left((\rho+1) \tilde{x}_{t}\right)\right]\right)-E\left[\exp \left(\tilde{x}_{t}\right)\right] E\left[\exp \left(\rho \tilde{x}_{t}\right)\right] \\
& =\exp \left(\frac{\sigma^{2}}{2}\right)\left(\exp \left(\frac{1}{2}(\rho+1)^{2} \sigma^{2}\left(\frac{1-\rho^{2 t}}{1-\rho^{2}}\right)\right)-\exp \left(\frac{1}{2} \sigma^{2}\left(\frac{1-\rho^{2 t}}{1-\rho^{2}}\right)\right) \exp \left(\frac{1}{2} \rho^{2} \sigma^{2}\left(\frac{1-\rho^{2 t}}{1-\rho^{2}}\right)\right)\right) \\
& =\exp \left(\frac{\sigma^{2}}{2}\right)\left(\exp \left(\frac{1}{2}\left(\rho^{2}+2 \rho+1\right) \sigma^{2}\left(\frac{1-\rho^{2 t}}{1-\rho^{2}}\right)\right)-\exp \left(\frac{1}{2}\left(\rho^{2}+1\right) \sigma^{2}\left(\frac{1-\rho^{2 t}}{1-\rho^{2}}\right)\right)\right) \\
& =\exp \left(\frac{\sigma^{2}}{2}\right) \exp \left(\frac{1}{2}\left(\rho^{2}+1\right) \sigma^{2}\left(\frac{1-\rho^{2 t}}{1-\rho^{2}}\right)\right)\left(\exp \left(\rho \sigma^{2}\left(\frac{1-\rho^{2 t}}{1-\rho^{2}}\right)\right)-1\right) \\
& =\exp \left(\frac{\sigma^{2}}{2}+\frac{1}{2}\left(\rho^{2}+1\right) \sigma^{2}\left(\frac{1-\rho^{2 t}}{1-\rho^{2}}\right)\right)\left(\exp \left(\rho \sigma^{2}\left(\frac{1-\rho^{2 t}}{1-\rho^{2}}\right)\right)-1\right) \\
& =\exp \left(\frac{\sigma^{2}}{2}\left(1+\left(\rho^{2}+1\right)\left(\frac{1-\rho^{2 t}}{1-\rho^{2}}\right)\right)\right)\left(\exp \left(\rho \sigma^{2}\left(\frac{1-\rho^{2 t}}{1-\rho^{2}}\right)\right)-1\right)
\end{aligned}
$$


Thus $\operatorname{Cov}\left(\tilde{\lambda}_{t}, \tilde{\lambda}_{t+1}\right)=\exp \left(\frac{\sigma^{2}}{2}\left(1+\left(\rho^{2}+1\right)\left(\frac{1-\rho^{2 t}}{1-\rho^{2}}\right)\right)\right)\left(\exp \left(\rho \sigma^{2}\left(\frac{1-\rho^{2 t}}{1-\rho^{2}}\right)\right)-1\right)$

Now, clearly, the first element is positive since it is an exponent. Further, since

$$
\sigma^{2}\left(\frac{1-\rho^{2 t}}{1-\rho^{2}}\right)>0
$$

we know that the second element is a strictly increasing function of $\rho$, reaching a value of zero at $\rho=0$. Therefore, for $\rho<0$, the expression is negative, while for $\rho>0$ the expression is positive. 